\title{
BRAINSTAT: A TOOLBOX FOR BRAIN-WIDE STATISTICS AND NEUROSCIENTIFIC CONTEXTUALIZATION
}

Reinder Vos de Wael ${ }^{1 *}$, Şeyma Bayrak ${ }^{2 *}$, Oualid Benkarim ${ }^{1}$, Peer Herholz ${ }^{1}$, Sara Larivière ${ }^{1}$, Raul Rodriguez-Cruces ${ }^{1}$, Casey Paquola ${ }^{3}$, Seok-Jun Hong ${ }^{4,5}$, Bratislav Misic ${ }^{1}$, Alan C. Evans ${ }^{1}$, Sofie L. Valk ${ }^{2,3 *}$, Boris C. Bernhardt ${ }^{1 *}$

*) Equal contributions

\author{
Affiliations \\ ${ }^{1}$ McConnell Brain Imaging Centre, Montreal Neurological Institute, McGill University, Montreal, Quebec, Canada; \\ ${ }^{2}$ Max Planck Institute for Human Cognitive and Brain Sciences, Leipzig, Germany; ${ }^{3}$ Institute of Neuroscience and \\ Medicine (INM-1), Forschungszentrum Jülich, Germany; ${ }^{4}$ Child Mind Institute, New York, USA; 5 Center for \\ Neuroscience Imaging Research, Institute for Basic Science, and Department of Biomedical Engineering, \\ Sungkyunkwan University, Suwon, South Korea
}

\section{Corresponding authors}

Boris C. Bernhardt, PhD

Montreal Neurological Institute

McGill University, Montreal, QC, Canada

Email: boris.bernhardt@mcgill.ca

Reinder Vos de Wael

Montreal Neurological Institute

McGill University, Montreal, QC, Canada

Email: reinder.vosdewael@mail.mcgill.ca 


\begin{abstract}
Analysis and interpretation of neuroimaging datasets has become a multidisciplinary endeavor, relying not only on statistical methods, but increasingly on associations with respect to other brain-derived features such as gene expression, histological data, and functional as well as cognitive architectures. Here we introduce BrainStat - a toolbox for (i) univariate and multivariate general linear models in volumetric and surface-based brain imaging datasets, and (ii) multidomain feature association of results with respect to spatial maps of post-mortem gene expression and histology, as well as task-based fMRI meta-analysis, canonical resting-state fMRI networks, and resting-state derived gradients across several common surface templates. The combination of statistics and feature associations into a turnkey toolbox offers to streamline analytical processes and accelerate cross-modal research. The toolbox is implemented in both Python and MATLAB, two widely used programming languages in the neuroimaging and neuroinformatics communities. BrainStat is openly available and complemented by an expandable documentation.
\end{abstract}

Key words: neuroimaging, multivariate analysis, univariate analysis 


\section{INTRODUCTION}

Neuroimaging enables brain-wide measures of morphology, microstructure, function, and connectivity in individuals as well as large cohorts (Casey et al., 2018; Glasser et al., 2013; Royer et al., 2021). Through an increasing array of powerful image processing techniques (Cameron et al., 2013; Esteban et al., 2019; Fischl, 2012; Kim et al., 2005), these data can nowadays be brought into a standardized frame of reference including stereotaxic voxel spaces, such as the commonly used MNI152 space (Collins et al., 2003; Dadar et al., 2018), surface-based space such as fsaverage, MNI152-CIVET surfaces or grayordinates spearheaded by the Human Connectome Project (Fischl, 2012; Glasser et al., 2013; Kim et al., 2005; Lyttelton et al., 2007; Marcus et al., 2011), as well as parcellation schemes (Desikan et al., 2006; Glasser et al., 2016; Gordon et al., 2016; Schaefer et al., 2017). Registering neuroimaging data to a common space allows for the application of statistical analyses, including mass-univariate generalized linear and mixed-effects models that carry out parallel statistical tests at each measurement unit. Usually, such analyses need to be carried out using multiple tools and programs, reducing the reproducibility of workflows, and increasing the risk of human error. With the current paper, we present BrainStat, a unified toolbox to implement these analyses in a cohesive and transparent framework.

The analytical workflows of neuroimaging studies increasingly rely on the availability of previously acquired datasets of other modalities. When mapped to the same reference frame as the neuroimaging measures, these datasets can be used for contextualization of findings and aid in interpretation and validation of results. For example, results may be contextualized within established motifs of the brain's functional architecture such as intrinsic functional communities based on resting-state fMRI (Yeo et al., 2011) or functional gradients (Margulies et al., 2016), both allowing for the interpretation of findings based on the established brain's functional architecture. Another common method for contextualization is automated meta-analysis with Neurosynth (Yarkoni et al., 2011), NiMARE (Salo et al., 2020), or BrainMap (Laird et al., 2005). These tools offer the ability to carry out ad hoc meta-analyses across potentially thousands of previously published fMRI studies. Correlating a statistical map with a database of brain activation maps related to cognitive terms, so-called meta-analytical decoding, offers a quantitative approach to infer plausible cognitive processes related to a spatial statistical pattern. Finally, post mortem datasets of transcriptomics (Hawrylycz et al., 2012) and histology (Amunts et al., 2013) mapped to a common neuroimaging space enable associations of neuroimaging findings with gene expression and histological patterns (Markello et al., 2021; Paquola et al., 2021). Such findings can provide information on molecular and cellular properties in the brain that spatially co-vary with an observed statistical map. By combining these feature association techniques, the functional, histological, and genetic correlates of 
neuroimaging findings can be inferred. BrainStat provides an integrated decoding engine to run these feature association techniques.

Our toolbox is implemented in both Python and MATLAB, two common programming languages in the neuroimaging research community. We have compartmentalized the toolbox into two main modules: statistics and contextualization. BrainStat relies on a simple object-oriented framework to streamline the analysis workflow. The toolbox is openly available at https://github.com/MICA-MNI/BrainStat with documentation available at https://brainstat.readthedocs.io/.

\section{RESULTS}

The main functionality of BrainStat is demonstrated through two tutorials. The first tutorial explains the statistics module, which allows for the implementation of linear models on neuroimaging data. The second tutorial showcases the context module, which computes feature associations with several published databases. Note that MATLAB live scripts and Jupyter notebooks tutorials of these modules are also available at https://brainstat.readthedocs.io/.

\section{Tutorial 1: Statistics Module}

Before describing the statistics module, we first provide a brief overview of general linear models (GLMs). GLMs take the form of $Y=X B+Z \gamma+e$, where $Y$ is an $n$-by- $m$ response matrix, $n$ is the number of samples (e.g., subjects), $m$ is the number of brain locations (e.g. voxels, vertices, parcels), $X$ and $Z$ are a fixed and random effects predictor matrices, respectively, $B$ and $\gamma$ are model coefficients of the fixed and random effects, respectively, and $e$ is a matrix of errors. Although fixed and random effects have been defined differently throughout the literature (Gelman, 2005), within BrainStat, fixed effects are assumed to be invariant for all groups within a population whereas random effects can differ between groups of a population. For example, consider an experiment where we model the effect of age on cortical thickness. If we assume that an additional year of age has the same effect on cortical thickness independent of whether the subject is 25 or 35 years old, then we may model this as a fixed effect. If we suspect that this relationship may differ across sexes, then we could add an additional fixed effect for sex as well as an interaction effect between age and sex. Baseline cortical thickness may also differ across individuals. To account for this, a random effect for subject could be added which allows for a random intercept for each subject, and an interaction effect between subject and age to allow for random slopes. Combining fixed and random effects allows GLMs to perform many common statistical procedures including: (M)AN(C)OVA, multiple regression, and repeated measures models (Friston, 2005; Nichols \& Holmes, 2002). As such, GLMs provide a powerful framework for statistical analysis. 
The statistics module of BrainStat is inspired by SurfStat (Chung et al., 2010; Worsley et al., 2009), a previously developed MATLAB package for the statistical analysis of neuroimaging data. The intent of the statistics module is to simplify the implementation of the general linear model and their multiple comparison corrections in neuroimaging data. The first step a user takes in the statistics module is to define the model. Given a user-specified $Y, X$, and $Z$, BrainStat may use one of two approaches to estimate model coefficients. If the model contains only fixed effects (i.e., the user provided only $Y$ and $X$ ), the problem boils down to solving an ordinary least squares problem for each brain location, with the coefficients computed as $B=\operatorname{pinv}(X) \cdot Y$, where $\operatorname{pinv}()$ denotes the Moore-Penrose pseudoinverse. BrainStat also accepts multivariate response data in fixed effects models (e.g., including thickness, curvature, T1w/T2w intensity). To do this, the user may provide $\mathrm{Y}$ as a $n$-by- $m$-by-variate matrix. In this case, model coefficients are estimated for each variate separately. If the user provides a mixed effects model (i.e., $Y, X$ and $Z$ are provided), then the coefficients are computed using a restricted maximum likelihood procedure (Chung, 2013). Variables of interest in a fixed or mixed model are defined in a contrast vector; this vector may be based on categorical variables (e.g., male/female), continuous variables (e.g., age) or interactions (e.g., age*sex). After defining the model and specifying the contrast interrogating the model, BrainStat computes the model test-statistics. If BrainStat is provided with univariate response data (e.g., cortical thickness), then a student t-test is used; if multivariate response data (e.g., curvature and cortical thickness) is provided, then Hotelling's T2 is used to determine the t-statistic. Lastly, the t-statistic may be used to derive p-values. BrainStat provides two options for multiple comparison corrections: false discovery rate (Benjamini \& Hochberg, 1995) and random field theory (Worsley et al., 1999). The false discovery rate procedure controls the proportion of false positives in the data, however it works only at the single-measurement level (i.e. vertex, voxel, parcel). Random field theory corrects for the probability of ever reporting a false positive. It allows for both peak-wise and cluster-wise p-values, however it requires data with a known topological structure (e.g., cortical surface; lattice derived from a volume).

To illustrate our previous example, we downloaded cortical thickness and demographic data of 70 participants, 12 of whom were scanned twice, of the MICs dataset (Royer et al., 2021) (Figure 1A). We ran a linear model with age and sex as well as their interaction effect as fixed effects, and subject as a random effect (Figure 1B). These are set up using the FixedEffect and MixedEffect (named as such as it may contain both random and fixed effects) classes. Next, we defined the contrast as -age, i.e., positive tvalues denote decreasing cortical thickness with age. This model was fitted on cortical thickness data using a one-tailed test. The t-values, clusterwise and peak p-values derived from random field theory, as well as the vertexwise p-values derived with a correction for false discovery rate were plotted in Figure 1C. We found that, in the MICs dataset, there is evidence for an effect of age on cortical thickness at the cluster level based on random-field theory, but no evidence for significant peaks within these clusters and little 
bioRxiv preprint doi: https://doi.org/10.1101/2022.01.18.476795; this version posted January 25, 2022. The copyright holder for this preprint (which was not certified by peer review) is the author/funder, who has granted bioRxiv a license to display the preprint in perpetuity. It is made available under aCC-BY 4.0 International license.

significance at a vertex-level. This suggests that the effect covers large regions, rather than local foci. Although we used a liberal cluster-defining threshold $(\mathrm{p}<0.01)$ for this illustration, we generally recommend a more stringent threshold $(\mathrm{p}<0.001)$ (Eklund et al., 2016; Woo et al., 2014). This example demonstrates how multiple levels of statistical complexity are supported by the BrainStat model.

A

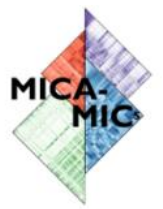

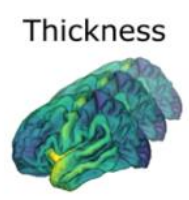

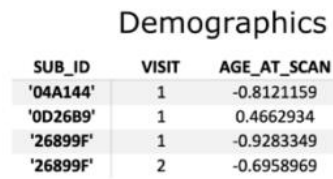

B

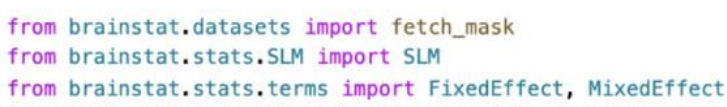

from brainstat.datasets import fetch_mask from brainstat.stats. SLM import SLM

from brainstat.stats.terms import FixedEffect, MixedEffect

from brainstat.tutorial.utils import fetch_mics_data

\# Load behavioral and cortical thickness data, and a midline mask.

thickness, demographics = fetch_mics_data( $)$

mask = fetch_mask ("fsaverage5")

\# Create a basic linear model.

fixed_age $=$ FixedEffect (demographics.AGE_AT_SCAN)

fixed_sex $=$ FixedEffect $($ demographics.SEX)

random_subject $=$ MixedEffect $($ demographics.SUB_ID)

model $=$ fixed_age + fixed_sex + fixed_age $*$ fixed_sex + random_subject

contrast_age $=-$ demographics.AGE_AT_SCAN.to_numpy $($ )

\# Fit the linear model

$\mathrm{slm}=$ SLM (

model,

contrast_age,

surf=" fsaverage5",

mask=mask,

correction= ["fdr", "rft"],

two_tailed=False,

)

cluster_threshold $=0.01$,

slm.fit (thickness)

C
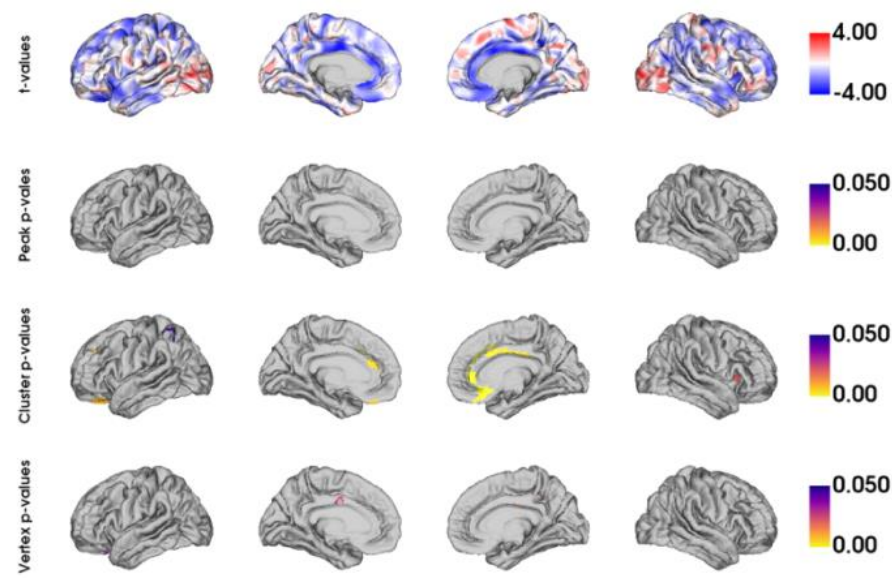

Figure 1. Example Python code for fitting a fixed effect general linear model of the effect of age on cortical thickness with BrainStat. (A) The MICS data included with BrainStat contains cortical thickness and demographics data. The demographics data contains hashed subject IDs (SUB_ID), visit number (VISIT), z-scored age on the day of scanning (AGE_AT_SCAN), and sex (SEX). (B) We create a linear model in the form of $Y=$ intercept + age. Note that the intercept is included in the model 
by default. Third, we initialize the model with an age contrast and request both p-values corrected with random field theory (i.e., "rft") as well as those corrected for false discovery rate (i.e., "fdr"). Lastly, we fit the model to the cortical thickness data. Equivalent MATLAB code can be found in Supplementary Figure 1 (C) Negative t-values (blue) indicate decreasing cortical thickness with age, whereas positive t-values (red) depict increasing cortical thickness with age. Significant peak-wise and cluster-wise $p$-values $(p<0.05)$ are shown for a random field theory (RFT) correction (cluster defining threshold $p<0.01$ ) as well as vertex-wise $p$-values $(\mathrm{p}<0.05)$ derived with false discovery rate $(\mathrm{FDR})$ correction. Code for plotting the figure can be found in Supplementary Figure 3.

\section{Tutorial 2: Context Module}

The context module bridges the gap between the statistical maps obtained from with the statistics module and external open-access datasets. As of version 0.3.3, this module consists of four submodules: $(i)$ transcriptomics/gene expression, (ii) task-based fMRI meta-analysis, (iii) resting-state fMRI connectomics, and (iv) post-mortem histology/cytoarchitecture. The transcriptomics submodule extracts genetic expression from the Allen Human Brain Atlas (Hawrylycz et al., 2012) for any given cortical parcellation. The meta-analytic submodule tests for associations between brain maps and meta-analytic task-fMRI maps associated with particular terms (Salo et al., 2020; Yarkoni et al., 2011). The resting-state module contextualizes neuroimaging findings using canonical networks (Yeo et al., 2011) and functional gradients (Margulies et al., 2016). The histological submodule fetches cell-body-staining intensity profiles from the BigBrain atlas (Amunts et al., 2013) at 50 different depths to extract cytoarchitectural axes (Paquola et al., 2019, 2021; Vos de Wael et al., 2020).

\section{Transcriptomics}

The Allen Human Brain Atlas (Hawrylycz et al., 2012) is a database of microarray gene expression data of over 20,000 genes derived from post-mortem tissue samples obtained from six adult donors. This resource may be used to derive associations between neuroimaging data and molecular factors (Arnatkeviciute, Fulcher, Bellgrove, et al., 2021) and thus yield insights into the mechanisms giving rise to anatomical and connectomic markers. For example, such data may be used to study associations between genetic factors and functional connectivity (Cioli et al., 2014; Krienen et al., 2016; Richiardi et al., 2015), anatomical connectivity (Goel et al., 2014; Park, Bethlehem, et al., 2021), as well as alterations of connectivity in disease (Park, Hong, et al., 2021; Romme et al., 2017). The genetic decoding module of BrainStat uses the abagen toolbox (Markello et al., 2021) to compute genetic expression data for a given parcellation. Using default parameters, abagen performs the following procedure. First it fetches and updates the MNI coordinates of tissue samples of all six donors using coordinates provided by the alleninf package (https://github.com/chrisgorgo/alleninf). Next, it performs an intensity-based filtering of the probes to remove those that do not exceed background noise. Subsequently, for probes indexing the same gene, it selects the probe with the highest differential stability across donors. The tissue samples are then matched 
to regions in the parcellation scheme. Expression values for each sample across genes and for each gene across samples are normalized using a scaled robust sigmoid normalization function. Lastly, samples within each region are averaged within each donor, then averaged across donors. For details on the procedures with non-default parameters please consult the abagen documentation (https://abagen.readthedocs.io/). In Python, BrainStat calls abagen directly, and as such all parameters may be modified. In MATLAB, where abagen is not available, we included genetic expression matrices precomputed with abagen with default parameters for many common parcellation schemes. In Figure 2 we show an example of fetching the genetic expression for a previously defined functional atlas (Schaefer et al., 2017), and correlating this to a the t-statistic map shown in Figure 1. The expression derived from this module can then be used in further analyses, for example by deriving the principal component of genetic expression and comparing it to previously derived statistical maps.

\section{A}
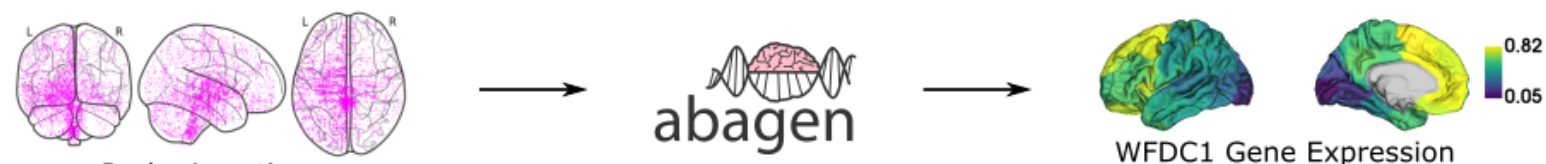

Probe Locations

B

import copy

import matplotlib.pyplot as plt

import numpy as np

import pandas as pd

from brainspace.utils.parcellation import reduce_by_labels

from matplotlib.cm import get_cmap

from brainstat. context.genetics import surface_genetic_expression

from brainstat.datasets import fetch_parcellation

\# Get Schaefer-100 genetic expression.

schaefer_100_fs5 = fetch_parcellation("fsaverage5", "schaefer", 100)

surfaces = fetch_template_surface ("fsaverage5", join=False)

expression = surface_genetic_expression (schaefer_100_fs5, surfaces, space=" fsaverage")

\# Plot Schaefer-100 genetic expression matrix.

colormap $=$ copy. $\operatorname{copy}($ get_cmap ()$)$

colormap.set_bad ( color="black")

plt.imshow(expression, aspect="auto", cmap=colormap)

plt. colorbar ().ax.tick_params ( labelsize $=14$ )

plt.xticks ( fontsize $=14$, rotation $=45$ )

plt. yticks ( fontsize=14)

plt.xlabel("Gene Index", fontdict=\{"fontsize": 16\})

plt.ylabel("Schaefer 100 Regions", fontdict=\{"fontsize": 16\})

plt.gcf ().subplots_adjust (bot tom $=0.2$ )

\#Plot correlation with WFDC1 gene

t_stat_schaefer_100 = reduce_by_labels (slm.t.flatten (), schaefer_100_fs5) [1: ]

$\mathrm{df}=$ pd.DataFrame $(\{" x ":$ t_stat_schaefer_100, "y": expression["WFDC1"] $\})$

df. dropna (inplace=True)

plt.scatter (df.x, df.y, $s=20, c=" k^{\prime \prime}$ )

plt.xticks ( fontsize $=14$ )

plt.yticks ( fontsize=14)

plt.xlabel("t-statistic", fontdict=\{"fontsize": 16\})

plt.ylabel("WFDC1 expression", fontdict $=\{$ "fontsize": 16\})

plt.plot(np.unique(df.x), np.polyld(np.polyfit(df.x, df.y, 1)) (np.unique(df.x)), "k")

plt.text $\left(-1.0,0.75, f^{\prime \prime} r=\{d f . x \cdot \operatorname{corr}(d f . y): .2 f\}^{\prime \prime}\right.$, fontdict $=\{"$ size": 14$\left.\}\right)$

WFDC1 Gene Expression
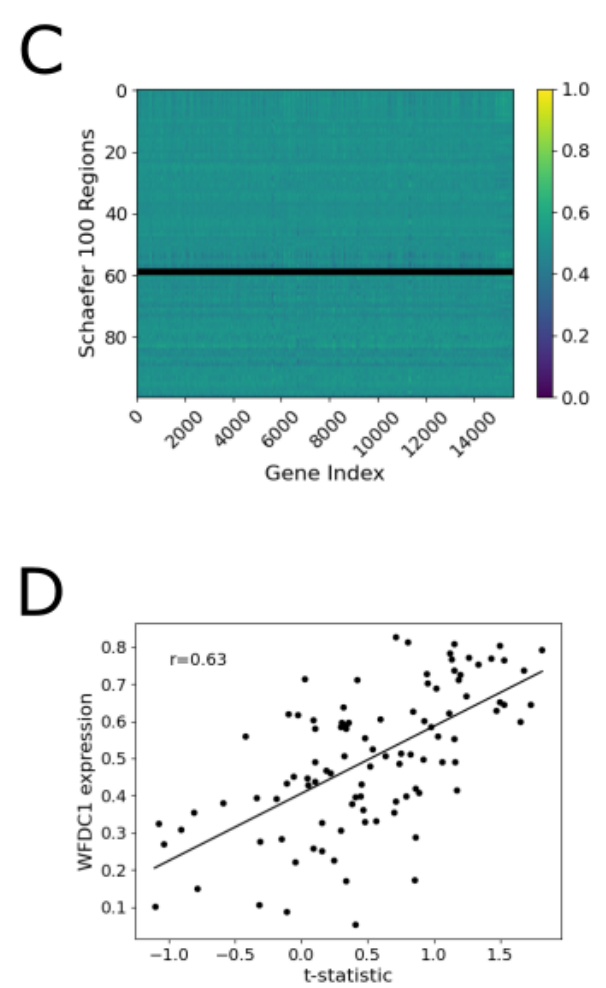

Figure 2. (A) BrainStat uses data from the Allen Human Brain atlas (Hawrylycz et al., 2012), provided by the Allen institute, and processed with abagen (Markello et al., 2021) to derive transcription levels of several thousands of genes. Shown are the 
locations of all probes, as well as the expression of a single gene (WFDC1) within 100 functionally defined regions (Schaefer et al., 2017). (B) Example Python code for computing the genetic expression based on a surface parcellation, plotting the matrix, and plotting a scatter plot with the WFDC1 gene. (C) The genetic expression matrix depicts the genetic expression, normalized to a range of $[0,1]$, of all parsed genes across all parcels. Black rows denote regions without samples. (D) Correlation between the t-statistic map plotted in Figure 1 and the WFDC1 gene expression. MATLAB code for retrieving and plotting genetic expression can be found in Supplementary Figure 5.

\section{Meta-analytic decoding}

The meta-analytic decoding submodule of BrainStat uses data derived from Neurosynth and NiMARE (Salo et al., 2020; Yarkoni et al., 2011) to decode a statistical map in terms of its cognitive underpinnings. In short, a meta-analytic activation map is created for many (cognitive) terms, and these maps may be correlated to a given statistical map to identify terms with the strongest relationship to the statistical map. This approach allows for the identification of indirect associations to cognitive terms used across a widerange of previously published task-based functional neuroimaging studies, without relying on cognitive tasks acquired in the same cohort. Indeed, meta-analytic decoding has been used by several groups to evaluate the cognitive associations of their neuroimaging findings [e.g., (Chang et al., 2013; Margulies et al., 2016; Paquola et al., 2019; Vogel et al., 2020; Vos de Wael et al., 2018)].

For each term in the Neurosynth database, we computed which studies used the term with a frequency of at least 1 in 1000 words (default parameter in NiMARE). Next, the meta-analytic maps were computed for these labels using a multilevel kernel density Chi-square analysis implemented in NiMARE (Salo et al., 2020; Wager et al., 2007). For any user-provided surface-based statistical map, we interpolate the map from surface space to volume space. Lastly, for every meta-analytic map in the database, using every voxel that exists within both the meta-analytic map as well as the statistical map we compute a product-moment correlation. An example of retrieving correlations with meta-analytic terms for the t-statistic map derived earlier (Figure 1) is shown in Figure 3. 

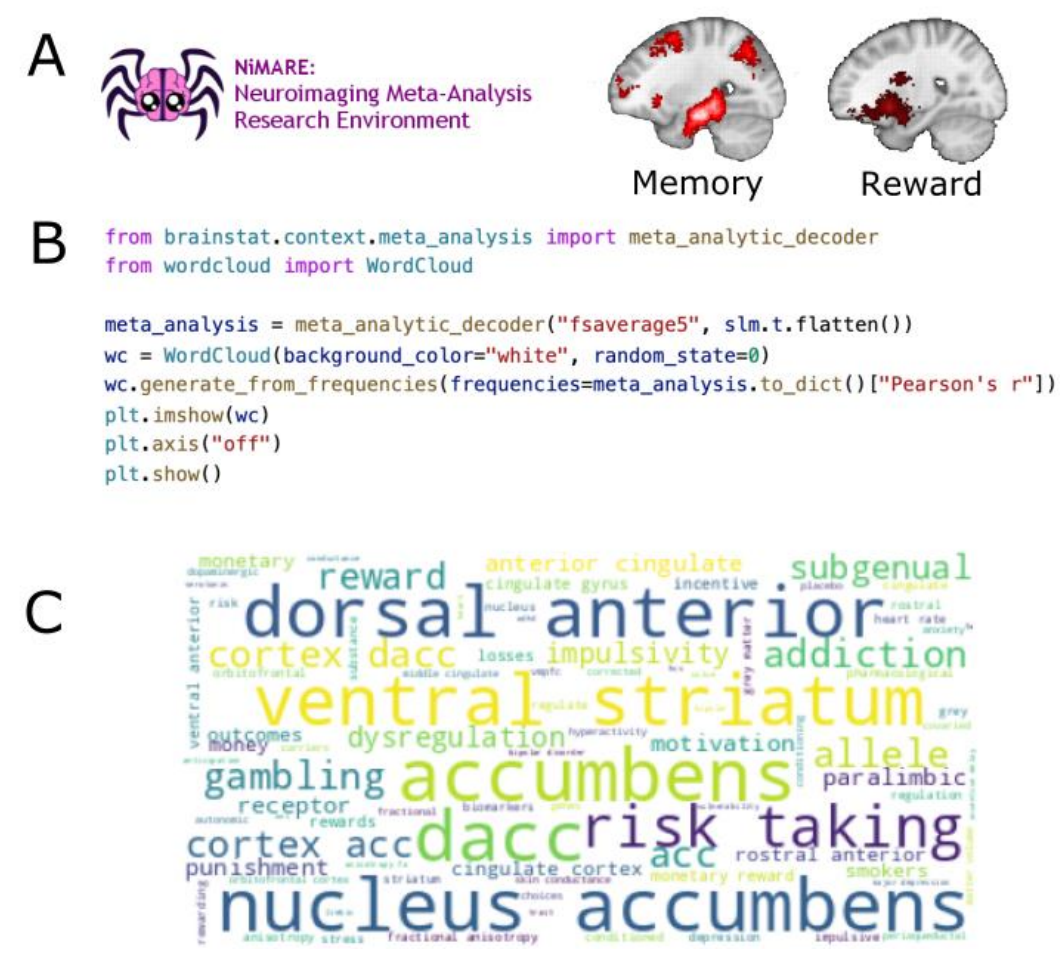

Figure 3. (A) Using the NiMARE toolbox (Salo et al., 2020) and the Neurosynth database (Yarkoni et al., 2011) we derived feature maps for every feature name in the Neurosynth database. We show an example association map for the terms "memory" and "reward". (B) Example Python code for computing the correlations between the t-statistics plotted in Figure 1 and every term in the Neurosynth database, and plotting these correlations as a word cloud. (C) The resultant word cloud from the code in

Figure 3B. Code for the MATLAB meta-analytic decoder can be found in Supplementary Figure 6.

\section{Resting-state motifs}

The functional architecture of the brain at rest has been described both as a set of discrete networks [e.g. (Smith et al., 2009; Yeo et al., 2011)] and as a set of continuous dimensions [also called gradients; (Margulies et al., 2016; Vos de Wael et al., 2020)]. The former highlights communities of regions with similar connectivity profiles, whereas the latter emphasizes gradual transitions between regions. Both approaches can be used to embed findings into the functional architecture of the human brain either by stratifying results in accordance with the functional architecture, or by assessing point-wise relationships to the gradients. Indeed, prior studies have assessed the relationship of the brain's functional architecture to cortical dynamics (Wang et al., 2019), high level cognition (Murphy et al., 2019; Shine et al., 2019; Sormaz et al., 2018), hippocampal subfield connectivity (Vos de Wael et al., 2018), amyloid beta expression and aging (Lowe et al., 2019), microstructural organization (Huntenburg et al., 2017; Paquola et al., 2019), phylogenetic changes (Xu et al., 2020), and alterations in disease states (Caciagli et al., 2021; Hong et al., 2019; Tian et al., 2019). 
The resting-state decoding module relies on two separate datasets: macroscopic networks as provided by Yeo, Krienen, et al. (Yeo et al., 2011), and functional gradients derived from the Human Connectome Project S1200 dataset (Van Essen et al., 2013). Associations of markers with the Yeo-Krienen networks are computed, for example, by taking the average of all data points within each network. Example code for computing the mean and standard error of the t-statistic map plotted in Figure 1 within the Yeo-Krienen networks are shown in Figure 4. The t-statistics are highest in the ventral attention and limbic networks, and lowest in the sensory regions. The functional gradients included with BrainStat were computed by resampling the mean functional connectivity matrix of the $\mathrm{S} 1200$ release to fsaverage5 (to reduce computational complexity), and subsequently computing gradients with BrainSpace using the following parameters: cosine similarity kernel, diffusion map embedding, alpha $=0.5$, sparsity $=0.9$, and diffusion_time $=0$ (Vos de Wael et al., 2020). Example code for computing the correlations between the first functional gradient and the t-statistic map plotted in Figure 1 are shown in Figure 5. We find a low correlation $(\mathrm{r}=0.17)$ between these two maps. However, to test for significance of this correlation we need to correct for the spatial autocorrelation in the data (Markello \& Misic, 2021). Three such corrections, namely spin test (Alexander-Bloch et al., 2018), Moran spectral randomization (Wagner \& Dray, 2015), and variogram matching (Burt et al., 2020), are included in BrainSpace (Vos de Wael et al., 2020), a dependency of BrainStat. Examples of how to compute the p-value for this correlation in Python (Supplementary Figure 9) and MATLAB (Supplementary Figure 10) are included in the supplemental materials.
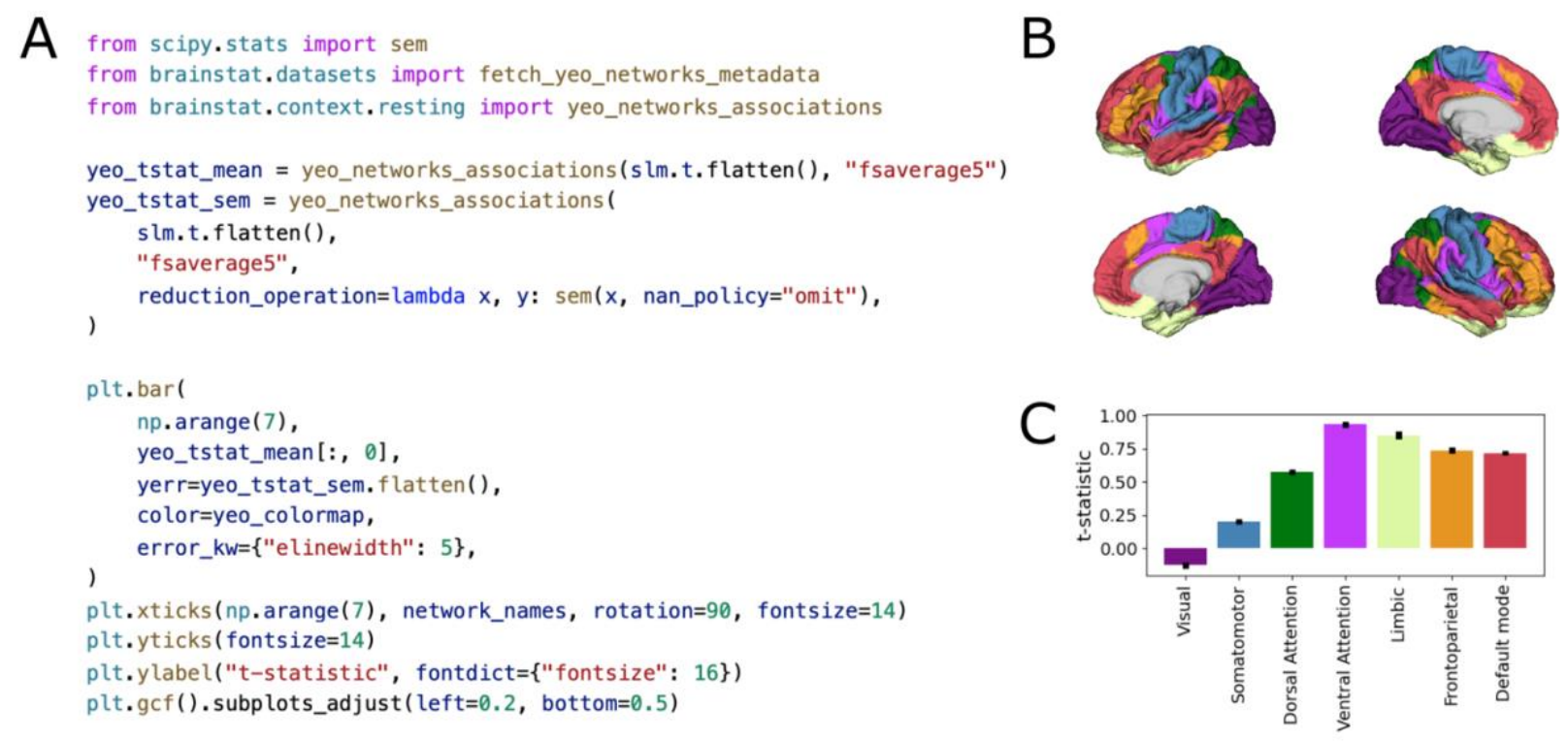


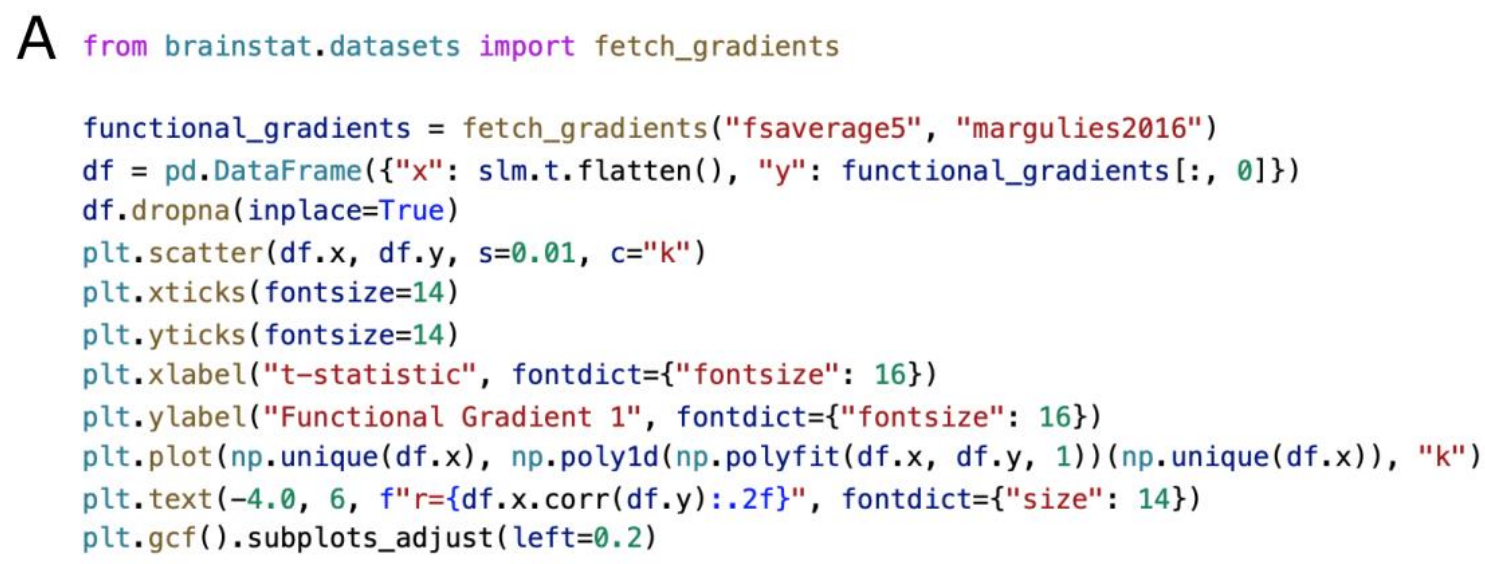

B

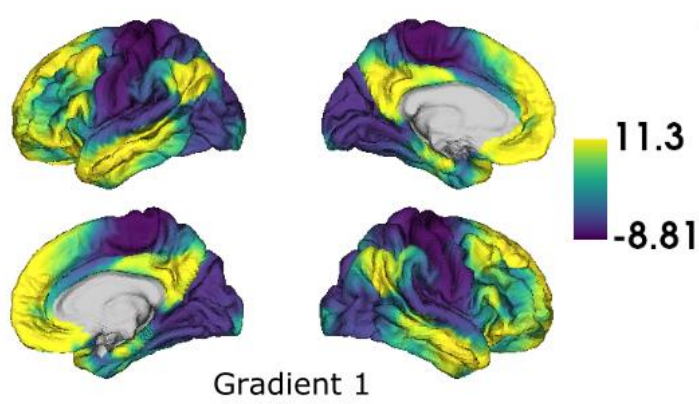

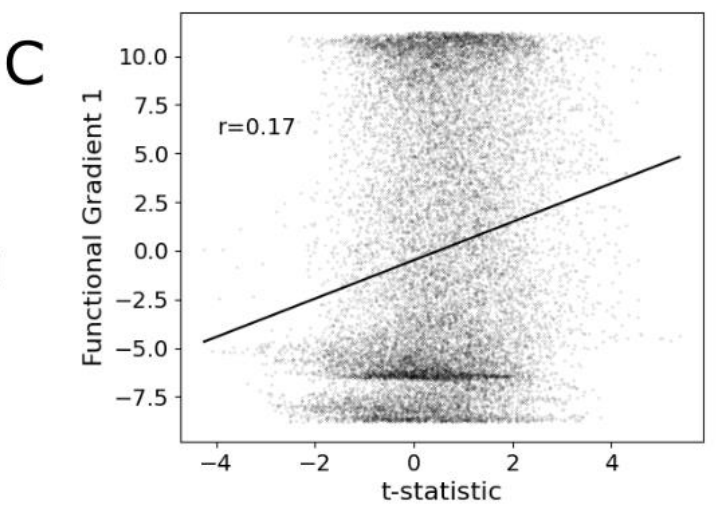

Figure 5. (A) Example Python code for computing the correlation of the t-statistic map plotted in Figure 1 and the first functional gradient, and plotting it in a scatter plot. Equivalent MATLAB code can be found in Supplementary Figure 8. (B) The first gradient of functional connectivity. (C) The scatter plot generated in (A).

Figure 4. (A) Example Python code for computing the mean and standard error of the mean of the t-statistic map plotted in Figure 1 within the Yeo-Krienen networks and plotting these in a bar plot. Equivalent MATLAB code can be found in Supplementary Figure 7. (B) The Yeo-Krienen networks plotted to the cortical surface. (C) The bar plot of the mean and standard error of the mean of the t-statistic map within the Yeo-Krienen networks.

\section{Histology}

The BigBrain atlas (Amunts et al., 2013) is a three-dimensional reconstruction of a sliced and cell-bodystained human brain. With a digitised isotropic resolution of 20 micrometers, it is the first openly available whole-brain 3D histological dataset. As such, it is well suited for relating neuroimaging markers to histological properties. This may be used, for example, for cross-validating MRI-derived microstructural findings (Paquola et al., 2019; Royer et al., 2020), defining regions of interest based on histological properties (Sitek et al., 2019), or relating connectomic markers to microstructure (Arnatkeviciute, Fulcher, Oldham, et al., 2021). The histology submodule aims to simplify the integration of neuroimaging findings with the BigBrain dataset. This submodule uses surfaces sampled from the BigBrain atlas at 50 different depths across the cortical mantle (Paquola et al., 2021). Covariance of these profiles, also known as 
microstructural profile covariance (Paquola et al., 2019), is computed with a partial correlation correcting for the mean intensity profile. Principal axes of cytoarchitectural variation are computed from microstructural profile covariance using BrainSpace with default parameters (Vos de Wael et al., 2020). An example of this is shown in Figure 6. We find a correlation between the first eigenvector and the tstatistic map of $r=-0.30$. Again, significance of this correlation should be tested with a correction for spatial autocorrelation as shown in Supplementary Figure 9 and Supplementary Figure 10.

A

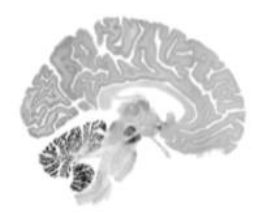

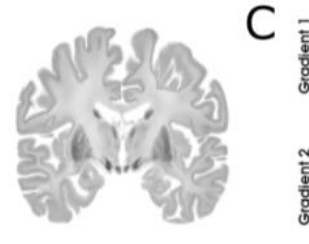

C

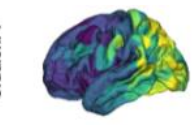

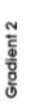

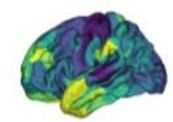

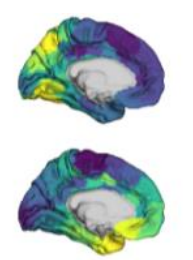
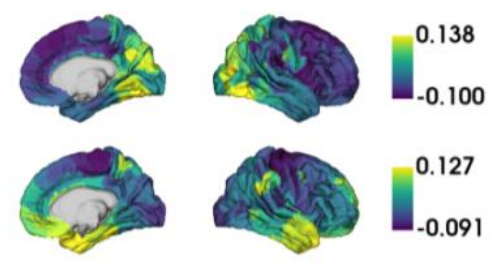

B

rom brainstat. context.histology import

compute_histology_gradients,

compute_mpc,

read_histology_profile,

\# Run the analysis.

schaefer_400 = fetch_parcellation ("fsaverage5", "schaefer", 400)

histology_profiles = read_histology_profile(template=" fsaverage $\left.5^{\prime \prime}\right)$

$\mathrm{mpc}=$ compute_mpc(histology_profiles, labels=schaefer_400)

gradient_map $=$ compute_histology_gradients $(m p c$, random_state $=0)$

\# Plot the correlation between the $t$-stat

t_stat_schaefer_400 $=$ reduce_by_labels $($ slm.t.flatten () , schaefer_400) [1: $]$

df $=$ pd.DataFrame $\left(\left\{" x^{\prime \prime}:\right.\right.$ t_stat_schaefer_400, "y": gradient_map.gradients_ $\left.\left.[:, 0]\right\}\right)$

df. dropna (inplace=True)

plt.scatter(df.x, df.y, s=5, c=" k")

plt. xticks ( fontsize=14)

plt.yticks ( fontsize $=14$ )

plt.xlabel("t-statistic", fontdict=\{"fontsize": 16$\})$

plt.ylabel("MPC Gradient 1", fontdict=\{"fontsize": 16\})

plt.plot(np.unique(df.x), np.poly1d(np.polyfit(df.x, df.y, 1)) (np.unique(df.x)), "k")

$p l t . t e x t(2.3,0.1, f " r=\{d f . x . c o r r(d f . y): .2 f\} "$, fontdict $=\{" s i z e ": 14\})$

plt.gcf().subplots_adjust ( left $=0.15$ )
D

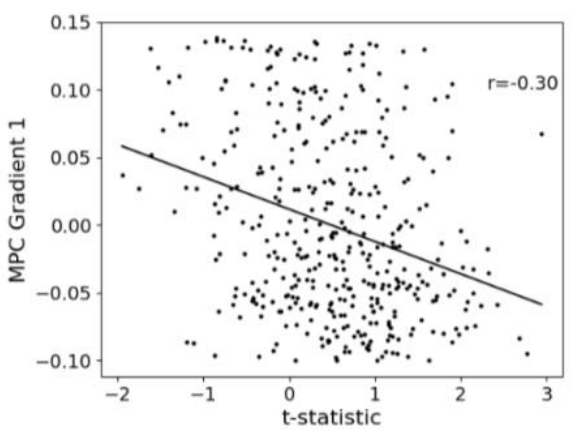

Figure 6. (A) Selected sagittal and coronal slices from the BigBrain atlas. (B) Example Python code for computing gradients of microstructural profile covariance for the Schaefer- 400 parcellation and correlating these to the t-statistic map plotted in Figure 1. Histology profiles, as computed in the BigBrainWarp toolbox, are loaded. From these profiles, a microstructural profile covariance matrix is built, and used to compute gradients of histology. The gradient and t-statistic map are correlated and plotted in a scatter plot. Equivalent MATLAB code can be found in Supplementary Figure 11. (C) Gradients derived from the microstructural profile covariance of the BigBrain atlas. (D) The scatter plot of the t-statistic map and the first gradient of microstructural profile covariance.

\section{Spatial-autocorrelative statistical tests}

The previous paragraphs have discussed multidomain feature associations between a user-provided statistical map and features derived from other databases. In most cases, users may wish to test whether these associations are statistically significant. Most standard statistical tests assume that measurements are independent of each other or have a known dependence structure. However, this assumption is violated in MRI data due to its spatial autocorrelation; spatially proximal measurements hold information on each other. Consequently, the effective degrees of freedom are lower than would be expected based on the 
number of measurements. To account for this, any statistical test should correct for this. This may be done by generating surrogate datasets that maintain the same spatial autocorrelation but are otherwise random. A statistic (e.g., correlation) may then be computed between the empirical/surrogate data and the predictor variable (e.g., functional gradient). The percentile of the empirical statistic within the distribution of surrogate statistics may then be used to compute the p-value. Several procedures have been proposed for this in recent years, most notably spin-test (Alexander-Bloch et al., 2018), Moran spectral randomization (Wagner \& Dray, 2015), and variogram matching (Burt et al., 2020). Spin-test requires that the data exists on a mesh with a corresponding sphere (e.g., fsaverage). The data is rotated on the sphere to create surrogate data. However, if large parts of the mesh contain no data, then spin-test will be unable to generate good surrogate data. To simplify the usage of spin-tests, spheres of several common surface templates are included with BrainStat in the function fetch_template_surface. Moran spectral randomization and variogram matching both quantify the spatial autocorrelation in the data and generate new data with similar spatial autocorrelation. These procedures are far more computationally expensive than spin-test, but they only require a distance metric between all pairs of measurements (e.g., Euclidean distance). Functions for all these spatial autocorrelation corrections are included with BrainSpace (Vos de Wael et al., 2020), a dependency of BrainStat (for examples see Supplementary Figure 9 and Supplementary Figure 10). Additionally, if multiple p-values are computed with corrections for spatial auto-correlation, then a correction for multiple comparisons [e.g., false discovery rate (Benjamini \& Hochberg, 1995)] should still be performed.

\section{DISCUSSION}

The analysis of brain imaging data demands tools for uni- and multivariate statistical inference, and increasingly leverages data from multiple resources to facilitate interpretation and contextualization of significant results. Although many tools have been made available by the neuroimaging community to perform these steps (Larivière et al., 2021; Markello et al., 2021, 2022; Paquola et al., 2021; Salo et al., 2020; Yarkoni et al., 2011), the integration of statistical inference and contextualization approaches currently requires in-house, often unpublished, tools. Here, we presented BrainStat, an integrated toolbox for the univariate and multivariate statistical analysis of brain imaging data and multidomain feature associations. The toolbox is programmed in both Python and MATLAB, two languages commonly used within the neuroimaging and network neurosciences communities. It is openly available through several hosting services, described in an expandable documentation complemented by accessible tutorials, and published under a license permissive of free modification, redistribution, and usage (BSD-3).

GLMs are a core technique to neuroimaging data analyses. Indeed, many common univariate and multivariate statistical analyses, including t-tests, F-tests, multiple linear regression, and (M)AN(C)OVA, 
can be considered special cases of the general linear model (Friston, 2005; Nichols \& Holmes, 2002). As such, usage of general linear models is widespread throughout the neuroimaging literature [e.g., (Bernhardt et al., 2018; Clarkson et al., 2011; Truong et al., 2013)]. Despite the prevalence of GLMs, their implementation is not trivial. BrainStat's statistics module aims to provide a flexible multivariate linear modeling framework for neuroimaging data (Worsley et al., 2009). The focus of this manuscript was to present the possibilities provided by BrainStat and outline an accessible tutorial for their usage within the toolbox. As such, we demonstrated a mixed effects models for testing the effects of age on cortical thickness. However, given the flexibility of GLMs, a large variety of models may be specified within the same framework. Furthermore, the flexible specification of a contrast simplifies interrogation of fitted models. Lastly, the included multiple comparisons corrections procedures, correction for false discovery rate (Benjamini \& Hochberg, 1995) and random field theory (Worsley et al., 1999), allow for inference both at the voxel/vertex level, as well as cluster-level inference. Whilst our examples were performed with fsaverage5, a commonly used surface template, the same statistical models can be applied to other surface spaces, volumetric data, parcellated data, and non-topographic data. As such, the statistics module constitutes a flexible tool able to run a wide variety of statistical models on neuroimaging data.

Recent years have seen an uptick in the usage of external datasets for the contextualization of MRI-derived results. These datasets may be leveraged for their unique advantages such as the unprecedented spatial resolution of the BigBrain histological atlas (Amunts et al., 2013), the vast number of studies included in the Neurosynth meta-analytical database (Yarkoni et al., 2011), and 3D maps of post-mortem human brain gene expression information aggregated in the Allen Human Brain Atlas (Hawrylycz et al., 2012). These external datasets allow for more comprehensive studies of brain organization and may advance our understanding of fundamental principles of brain organization (Hansen et al., 2021; Larivière et al., 2019). Indeed, prior studies have used these datasets to relate task meta-analyses, genetic expression, and histology to markers of morphology (Valk et al., 2020; Wagstyl et al., 2020; Whitaker et al., 2016), function (Benkarim et al., 2021; Krienen et al., 2016; Margulies et al., 2016; Paquola et al., 2019), and structural connectivity (Romme et al., 2017; Vos de Wael et al., 2021). Though numerous packages exist to enable these analyses (Markello et al., 2021; Paquola et al., 2021; Salo et al., 2020; Yarkoni et al., 2011), using and integrating these requires expertise and proficiency in their respective programming languages. BrainStat brings these tools together into a unified multi-language framework, thereby increasing their accessibility and streamlining the analytics processes of neuroimaging studies. Ultimately, we hope to reduce the barrier to entry of these techniques, reduce the chances of human error, and thereby accelerate cross-modal research in the neuroimaging community. 
The context module provides a powerful framework to bridge gaps between microscale features and macroscale brain organization. However, any analyses performed with this module are subject to the limitations of the dataset used, as well as the limitations of cross-dataset comparisons. The Allen Human Brain Atlas and the BigBrain atlas consist of only six and one post-mortem subjects, respectively (Amunts et al., 2013; Hawrylycz et al., 2012). The functional gradients and networks included with BrainStat were all derived from neurotypical young adults (Van Essen et al., 2013; Yeo et al., 2011). As such, these data may not be guaranteed to generalize well to other age groups, or individuals with atypical brain organization. The Neurosynth database relies on the reporting of peak coordinates, as such its spatial resolution is limited by the resolution of its studies and the extent of clusters is unknown (Yarkoni et al., 2011). Furthermore, none of these datasets may be manipulated by experimental design, as such no causal inference can be drawn. Despite these limitations, this module allows researchers to access microscale and macroscale brain features without incurring the costs of data acquisition. As such, the context module may serve three purposes in common data analyses: aiding in interpretation of results, exploratory analysis, and providing additional support for a priori hypotheses.

Theoretical and empirical studies have shown the importance of replicability in science (Ioannidis, 2005; Moonesinghe et al., 2007; Open Science Collaboration, 2015). The proliferation of open-access datasets (Di Martino et al., 2014; Miller et al., 2016; Royer et al., 2021; Van Essen et al., 2013) and software (Fischl, 2012; Marcus et al., 2011; Paquola et al., 2021; Tournier et al., 2012; Vos de Wael et al., 2020) may increase reproducibility by allowing others to redo experiments with identical data and procedures, as well as reducing human error in the analysis (Milham et al., 2018; Poldrack et al., 2017). BrainStat may contribute to this process. By unifying statistical processing and multidomain feature association into a single package across two programming languages, the resulting code will require less customization and technical expertise from the end-user. Furthermore, BrainStat may increase the accessibility to all these methods for researchers in places that lack the institutional expertise to set up such integrated pipelines.

\section{METHODS}

\section{$\underline{\text { Tutorial dataset }}$}

We studied 70 healthy participants $[30$ females, age $=31.9 \pm 8.9$ (mean $+\mathrm{SD}) ; 12$ of them ( 5 female) came in for a second visit with age $=32.8 \pm 7.5$ ] of the MICS dataset (Royer et al., 2021). Note that these data include subjects not part of the current MICS release. For each visit, two T1w images were derived with the following parameters: MP-RAGE; 0.8mm isotropic voxels, matrix $=320 \times 320,224$ sagittal slices, TR $=2300 \mathrm{~ms}, \mathrm{TE}=3.14 \mathrm{~ms}, \mathrm{TI}=900 \mathrm{~ms}$, flip angle $=9^{\circ}, \mathrm{PAT}=2$, partial Fourier $=6 / 8$. Scans were visually inspected for motion artefacts. Processing was performed with micapipe (https://github.com/MICA$\mathrm{MNI} /$ micapipe). In short, cortical surface segmentations were generated from the $\mathrm{T} 1 \mathrm{w}$ scans using 
Freesurfer 6.0 (Fischl, 2012). Subject's thickness estimates were transformed to the fsaverage5 template and smoothed with a $10 \mathrm{~mm}$ full-width-at-half-maximum Gaussian kernel.

\section{Code Availability}

BrainStat is freely available through PyPi (https://pypi.org/project/brainstat/; installable with 'pip install brainstat ${ }^{\prime}$ ), FileExchange (https://www.mathworks.com/matlabcentral/fileexchange/89827-brainstat; installable with the add-on manager in MATLAB), and GitHub (https://github.com/MICA-MNI/BrainStat). Documentation is available at https://brainstat.readthedocs.io/. At the time of writing (BrainStat version 0.3.3), BrainStat supports Python 3.7-3.9 and MATLAB R2019b+ on Windows, macOS, and Linux. However, we advise users to consult the installation guide in our documentation for up-to-date requirements.

\section{ACKNOWLEDGMENTS}

The authors would like to thank the late Keith Worsley for his invaluable work on the SurfStat toolbox which inspired BrainStat.

\section{AUTHOR CONTRIBUTIONS}

R.V., and Ş.B. were the lead developers of the toolbox. O.B., P.H., S.V, and B.B. further assisted in toolbox design. R.V., and B.B. drafted the manuscript. All authors assisted in revising the manuscript.

\section{COMPETING INTERESTS STATEMENT}

All authors declare no competing interests.

\section{FUNDING}

R.V. was funded by the Richard and Ann Sievers award. O.B. was funded by a Healthy Brains for Healthy Lives (HBHL) postdoctoral fellowship and the Quebec Autism Research Training (QART) program. P.H. was supported in parts by funding from the Canada First Research Excellence Fund, awarded to McGill University for the Healthy Brains for Healthy Lives initiative, the National Institutes of Health (NIH) NIH-NIBIB P41 EB019936 (ReproNim), the National Institute Of Mental Health of the NIH under Award Number R01MH096906, a research scholar award from Brain Canada, in partnership with Health Canada, for the Canadian Open Neuroscience Platform initiative, as well as an Excellence Scholarship from Unifying Neuroscience and Artificial Intelligence - Québec. S.L. acknowledges funding from CIHR and the Richard and Ann Sievers award. RRC received support from the Fonds de la Recherche du Québec - Santé (FRQ-S). C.P. was funded by 
Helmholtz International BigBrain Analytics Learning Laboratory (HIBALL). S.J.H. was funded by was supported by funding from the Brain \& Behavior Research Foundation (NARSAD Young Investigator grant; \#28436) and the Institute for Basic Science (IBS-R15-D1) in Korea. B.M. acknowledges support from the Natural Sciences and Engineering Research Council of Canada (NSERC), the Canadian Institutes of Health Research (CIHR), the Canda Research Chairs Program (CRC), Brain Canada Future Leaders, and the Healthy Brains Healthy Lives initiative (HBHL). S.L.V. acknowledges support from the Otto Hahn award from the Max Planck society and Helmholtz International BigBrain Analytics and Learning Laboratory (HIBALL). B.B. acknowledges research support from the National Science and Engineering Research Council of Canada (NSERC Discovery-1304413), the CIHR (FDN-154298, PJT174995), SickKids Foundation (NI17-039), Azrieli Center for Autism Research (ACARTACC), BrainCanada, FRQS, the Tier-2 Canada Research Chairs program. A.E. and B.B. acknowledge funding from the Helmholtz International BigBrain Analytics and Learning Laboratory (HIBALL).

\section{REFERENCES}

Alexander-Bloch, A. F., Shou, H., Liu, S., Satterthwaite, T. D., Glahn, D. C., Shinohara, R. T., Vandekar, S. N., \& Raznahan, A. (2018). On testing for spatial correspondence between maps of human brain structure and function. NeuroImage. https://doi.org/10.1016/j.neuroimage.2018.05.070

Amunts, K., Lepage, C., Borgeat, L., Mohlberg, H., Dickscheid, T., Rousseau, M.-É., Bludau, S., Bazin, P.-L., Lewis, L. B., Oros-Peusquens, A.-M., \& others. (2013). BigBrain: An ultrahigh-resolution 3D human brain model. Science, 340(6139), 1472-1475.

Arnatkeviciute, A., Fulcher, B. D., Bellgrove, M. A., \& Fornito, A. (2021). Imaging transcriptomics of brain disorders. Biological Psychiatry Global Open Science. https://doi.org/10.1016/j.bpsgos.2021.10.002

Arnatkeviciute, A., Fulcher, B. D., Oldham, S., Tiego, J., Paquola, C., Gerring, Z., Aquino, K., Hawi, Z., Johnson, B., Ball, G., Klein, M., Deco, G., Franke, B., Bellgrove, M. A., \& Fornito, A. (2021). Genetic influences on hub connectivity of the human connectome. Nature Communications, 12(1), 4237. https://doi.org/10.1038/s41467-021-24306-2

Benjamini, Y., \& Hochberg, Y. (1995). Controlling the False Discovery Rate. J. Royal Stat. Soc. B, 57, 2890399. 
Benkarim, O., Paquola, C., Park, B., Hong, S.-J., Royer, J., Vos de Wael, R., Lariviere, S., Valk, S., Bzdok, D., Mottron, L., \& others. (2021). Connectivity alterations in autism reflect functional idiosyncrasy. Communications Biology, 4(1), 1-15.

Bernhardt, B. C., Fadaie, F., Vos de Wael, R., Hong, S. J., Liu, M., Guiot, M. C., Rudko, D. A., Bernasconi, A., \& Bernasconi, N. (2018). Preferential susceptibility of limbic cortices to microstructural damage in temporal lobe epilepsy: A quantitative T1 mapping study. NeuroImage, 182, 294-303. https://doi.org/10.1016/j.neuroimage.2017.06.002

Burt, J. B., Helmer, M., Shinn, M., Anticevic, A., \& Murray, J. D. (2020). Generative modeling of brain maps with spatial autocorrelation. NeuroImage, 220, 117038. https://doi.org/10.1016/j.neuroimage.2020.117038

Caciagli, L., Paquola, C., He, X., Vollmar, C., Centeno, M., Wandschneider, B., Braun, U., Trimmel, K., Vos, S. B., Sidhu, M. K., \& others. (2021). Disorganization of language and working memory systems in frontal versus temporal lobe epilepsy. MedRxiv.

Cameron, C., Sharad, S., Brian, C., Ranjeet, K., Satrajit, G., Chaogan, Y., Qingyang, L., Daniel, L., Joshua, V., Randal, B., Stanley, C., Maarten, M., Clare, K., Adriana, D. M., Francisco, C., \& Michael, M. (2013). Towards Automated Analysis of Connectomes: The Configurable Pipeline for the Analysis of Connectomes (C-PAC). Frontiers in Neuroinformatics, 7. https://doi.org/10.3389/conf.fninf.2013.09.00042

Casey, B. J., Cannonier, T., Conley, M. I., Cohen, A. O., Barch, D. M., Heitzeg, M. M., Soules, M. E., Teslovich, T., Dellarco, D. V., Garavan, H., Orr, C. A., Wager, T. D., Banich, M. T., Speer, N. K., Sutherland, M. T., Riedel, M. C., Dick, A. S., Bjork, J. M., Thomas, K. M., .. Dale, A. M. (2018). The Adolescent Brain Cognitive Development (ABCD) study: Imaging acquisition across 21 sites. Developmental Cognitive Neuroscience, 32, 43-54. https://doi.org/10.1016/j.dcn.2018.03.001

Chang, L. J., Yarkoni, T., Khaw, M. W., \& Sanfey, A. G. (2013). Decoding the Role of the Insula in Human Cognition: Functional Parcellation and Large-Scale Reverse Inference. Cerebral Cortex, 23(3), 739-749. https://doi.org/10.1093/cercor/bhs065 
Chung, M. K. (2013). Computational neuroanatomy: The methods. World Scientific.

Chung, M. K., Worsley, K. J., Nacewicz, B. M., Dalton, K. M., \& Davidson, R. J. (2010). General multivariate linear modeling of surface shapes using SurfStat. NeuroImage, 53(2), 491-505. https://doi.org/10.1016/j.neuroimage.2010.06.032

Cioli, C., Abdi, H., Beaton, D., Burnod, Y., \& Mesmoudi, S. (2014). Differences in Human Cortical Gene Expression Match the Temporal Properties of Large-Scale Functional Networks. PLOS ONE, 9(12), e115913. https://doi.org/10.1371/journal.pone.0115913

Clarkson, M. J., Cardoso, M. J., Ridgway, G. R., Modat, M., Leung, K. K., Rohrer, J. D., Fox, N. C., \& Ourselin, S. (2011). A comparison of voxel and surface based cortical thickness estimation methods. NeuroImage, 57(3), 856-865. https://doi.org/10.1016/j.neuroimage.2011.05.053

Collins, D. L., Zijdenbos, A. P., Paus, T., \& Evans, A. C. (2003). Use of registration for cohort studies. Medical Image Registration.

Dadar, M., Fonov, V. S., Collins, D. L., Initiative, A. D. N., \& others. (2018). A comparison of publicly available linear MRI stereotaxic registration techniques. Neuroimage, 174, 191-200.

Desikan, R. S., Ségonne, F., Fischl, B., Quinn, B. T., Dickerson, B. C., Blacker, D., Buckner, R. L., Dale, A. M., Maguire, R. P., Hyman, B. T., \& others. (2006). An automated labeling system for subdividing the human cerebral cortex on MRI scans into gyral based regions of interest. Neuroimage, 31(3), 968-980.

Di Martino, A., Yan, C.-G., Li, Q., Denio, E., Castellanos, F. X., Alaerts, K., Anderson, J. S., Assaf, M., Bookheimer, S. Y., Dapretto, M., Deen, B., Delmonte, S., Dinstein, I., Ertl-Wagner, B., Fair, D. A., Gallagher, L., Kennedy, D. P., Keown, C. L., Keysers, C., ... Milham, M. P. (2014). The autism brain imaging data exchange: Towards a large-scale evaluation of the intrinsic brain architecture in autism. Molecular Psychiatry, 19(6), 659-667. https://doi.org/10.1038/mp.2013.78

Eklund, A., Nichols, T. E., \& Knutsson, H. (2016). Cluster failure: Why fMRI inferences for spatial extent have inflated false-positive rates. Proceedings of the National Academy of Sciences, 113(28), 79007905. https://doi.org/10.1073/pnas.1602413113 
Esteban, O., Markiewicz, C. J., Blair, R. W., Moodie, C. A., Isik, A. I., Erramuzpe, A., Kent, J. D., Goncalves, M., DuPre, E., Snyder, M., Oya, H., Ghosh, S. S., Wright, J., Durnez, J., Poldrack, R. A., \& Gorgolewski, K. J. (2019). fMRIPrep: A robust preprocessing pipeline for functional MRI. Nature Methods, 16(1), 111-116. https://doi.org/10.1038/s41592-018-0235-4

Fischl, B. (2012) FreeSurfer.

Neuroimage, $62(2)$ $774-781$. https://doi.org/10.1016/j.neuroimage.2012.01.021

Friston, K. J. (2005). Models of Brain Function in Neuroimaging. Annual Review of Psychology, 56(1), 5787. https://doi.org/10.1146/annurev.psych.56.091103.070311

Gelman, A. (2005). Analysis of variance-Why it is more important than ever. The Annals of Statistics, $33(1), 1-53$.

Glasser, M. F., Coalson, T. S., Robinson, E. C., Hacker, C. D., Harwell, J., Yacoub, E., Ugurbil, K., Andersson, J., Beckmann, C. F., Jenkinson, M., \& others. (2016). A multi-modal parcellation of human cerebral cortex. Nature, 536(7615), 171. https://doi.org/10.1038/nature18933

Glasser, M. F., Sotiropoulos, S. N., Wilson, J. A., Coalson, T. S., Fischl, B., Andersson, J. L., Xu, J., Jbabdi, S., Webster, M., Polimeni, J. R., Van Essen, D. C., \& Jenkinson, M. (2013). The minimal preprocessing pipelines for the Human Connectome Project. NeuroImage, 80, 105-124. https://doi.org/10.1016/j.neuroimage.2013.04.127

Goel, P., Kuceyeski, A., LoCastro, E., \& Raj, A. (2014). Spatial patterns of genome-wide expression profiles reflect anatomic and fiber connectivity architecture of healthy human brain. Human Brain Mapping, 35(8), 4204-4218.

Gordon, E. M., Laumann, T. O., Adeyemo, B., Huckins, J. F., Kelley, W. M., \& Petersen, S. E. (2016). Generation and evaluation of a cortical area parcellation from resting-state correlations. Cerebral Cortex, 26(1), 288-303.

Hansen, J. Y., Markello, R. D., Vogel, J. W., Seidlitz, J., Bzdok, D., \& Misic, B. (2021). Mapping gene transcription and neurocognition across human neocortex. Nature Human Behaviour, 5(9), 12401250. https://doi.org/10.1038/s41562-021-01082-z 
Hawrylycz, M. J., Lein, E. S., Guillozet-Bongaarts, A. L., Shen, E. H., Ng, L., Miller, J. A., van de Lagemaat, L. N., Smith, K. A., Ebbert, A., Riley, Z. L., Abajian, C., Beckmann, C. F., Bernard, A., Bertagnolli, D., Boe, A. F., Cartagena, P. M., Chakravarty, M. M., Chapin, M., Chong, J., ... Jones, A. R. (2012). An anatomically comprehensive atlas of the adult human brain transcriptome. Nature, 489(7416), 391-399. https://doi.org/10.1038/nature11405

Hong, S.-J., Vos de Wael, R., Bethlehem, R. A. I., Lariviere, S., Paquola, C., Valk, S. L., Milham, M. P., Di Martino, A., Margulies, D. S., Smallwood, J., \& Bernhardt, B. C. (2019). Atypical functional $\begin{array}{lllll}\text { connectome hierarchy in autism. Nature Communications, } 10(1), & 1022 .\end{array}$ https://doi.org/10.1038/s41467-019-08944-1

Huntenburg, J. M., Bazin, P. L., Goulas, A., Tardif, C. L., Villringer, A., \& Margulies, D. S. (2017). A Systematic Relationship Between Functional Connectivity and Intracortical Myelin in the Human Cerebral Cortex. Cerebral Cortex (New York, N.Y.: 1991), 27(2), 981-997. https://doi.org/10.1093/cercor/bhx030

Ioannidis, J. P. (2005). Why most published research findings are false. PLoS Medicine, 2(8), e124.

Kim, J., Singh, V., Lee, J., Lerch, J., Ad-Dab'bagh, Y., MacDonald, D., Lee, J., Kim, S., \& Evans, A. (2005). Automated 3-D extraction and evaluation of the inner and outer cortical surfaces using a Laplacian map and partial volume effect classification. Neuroimage, 27(1), 210-221.

Krienen, F. M., Yeo, B. T. T., Ge, T., Buckner, R. L., \& Sherwood, C. C. (2016). Transcriptional profiles of supragranular-enriched genes associate with corticocortical network architecture in the human brain. Proceedings of the National Academy of Sciences, 113(4), E469-E478. https://doi.org/10.1073/pnas.1510903113

Laird, A. R., Lancaster, J. J., \& Fox, P. T. (2005). Brainmap. Neuroinformatics, 3(1), 65-77.

Larivière, S., Paquola, C., Park, B., Royer, J., Wang, Y., Benkarim, O., Vos de Wael, R., Valk, S. L., Thomopoulos, S. I., Kirschner, M., \& others. (2021). The ENIGMA Toolbox: Multiscale neural contextualization of multisite neuroimaging datasets. Nature Methods, 18(7), 698-700. 
Larivière, S., Vos de Wael, R., Paquola, C., Hong, S.-J., Mišić, B., Bernasconi, N., Bernasconi, A., Bonilha, L., \& Bernhardt, B. C. (2019). Microstructure-Informed Connectomics: Enriching Large-Scale Descriptions of Healthy and Diseased Brains. Brain Connectivity, 9(2), 113-127. https://doi.org/10.1089/brain.2018.0587

Lowe, A. J., Paquola, C., Vos de Wael, R., Girn, M., Lariviere, S., Tavakol, S., Caldairou, B., Royer, J., Schrader, V. D., Bernasconi, A., Bernasconi, N., Spreng, R. N., \& Bernhardt, B. C. (2019). Targeting age-related differences in brain and cognition with multimodal imaging and connectome topography profiling. Human Brain Mapping, 40(18), 5213-5230. https://doi.org/10.1002/hbm.24767

Lyttelton, O., Boucher, M., Robbins, S., \& Evans, A. (2007). An unbiased iterative group registration template for cortical surface analysis. Neuroimage, 34(4), 1535-1544.

Marcus, D., Harwell, J., Olsen, T., Hodge, M., Glasser, M., Prior, F., Jenkinson, M., Laumann, T., Curtiss, S., \& Van Essen, D. (2011). Informatics and data mining tools and strategies for the human connectome project. Frontiers in Neuroinformatics, 5, 4.

Margulies, D. S., Ghosh, S. S., Goulas, A., Falkiewicz, M., Huntenburg, J. M., Langs, G., Bezgin, G., Eickhoff, S. B., Castellanos, F. X., Petrides, M., Jefferies, E., \& Smallwood, J. (2016). Situating the default-mode network along a principal gradient of macroscale cortical organization. Proc. Natl. Acad. Sci. U. S. A., 113(44), 12574-12579. https://doi.org/10.1073/pnas.1608282113

Markello, R. D., Arnatkeviciute, A., Poline, J.-B., Fulcher, B. D., Fornito, A., \& Misic, B. (2021). Standardizing workflows in imaging transcriptomics with the abagen toolbox. ELife, 10, e72129. https://doi.org/10.7554/eLife.72129

Markello, R. D., Hansen, J. Y., Liu, Z.-Q., Bazinet, V., Shafiei, G., Suarez, L. E., Blostein, N., Seidlitz, J., Baillet, S., Satterthwaite, T. D., Chakravarty, M., Raznahan, A., \& Misic, B. (2022). Neuromaps: Structural and functional interpretation of brain maps (p. 2022.01.06.475081). https://doi.org/10.1101/2022.01.06.475081 
Markello, R. D., \& Misic, B. (2021). Comparing spatial null models for brain maps. NeuroImage, 236, 118052.

Milham, M. P., Craddock, R. C., Son, J. J., Fleischmann, M., Clucas, J., Xu, H., Koo, B., Krishnakumar, A., Biswal, B. B., Castellanos, F. X., Colcombe, S., Di Martino, A., Zuo, X.-N., \& Klein, A. (2018). Assessment of the impact of shared brain imaging data on the scientific literature. Nature Communications, 9(1), 2818. https://doi.org/10.1038/s41467-018-04976-1

Miller, K. L., Alfaro-Almagro, F., Bangerter, N. K., Thomas, D. L., Yacoub, E., Xu, J., Bartsch, A. J., Jbabdi, S., Sotiropoulos, S. N., Andersson, J. L., Griffanti, L., Douaud, G., Okell, T. W., Weale, P., Dragonu, I., Garratt, S., Hudson, S., Collins, R., Jenkinson, M., ... Smith, S. M. (2016). Multimodal population brain imaging in the UK Biobank prospective epidemiological study. Nature Neuroscience, 19(11), 1523-1536. https://doi.org/10.1038/nn.4393

Moonesinghe, R., Khoury, M. J., \& Janssens, A. C. J. W. (2007). Most Published Research Findings Are False-But a Little Replication Goes a Long Way. PLOS Medicine, 4(2), e28. https://doi.org/10.1371/journal.pmed.0040028

Murphy, C., Wang, H.-T., Konu, D., Lowndes, R., Margulies, D. S., Jefferies, E., \& Smallwood, J. (2019). Modes of operation: A topographic neural gradient supporting stimulus dependent and independent cognition. NeuroImage, 186, 487-496.

Nichols, T. E., \& Holmes, A. P. (2002). Nonparametric permutation tests for functional neuroimaging: A primer with examples. Human Brain Mapping, 15(1), 1-25.

Open Science Collaboration. (2015). Estimating the reproducibility of psychological science. Science, $349(6251)$.

Paquola, C., Royer, J., Lewis, L. B., Lepage, C., Glatard, T., Wagstyl, K., DeKraker, J., Toussaint, P.-J., Valk, S. L., Collins, L., Khan, A. R., Amunts, K., Evans, A. C., Dickscheid, T., \& Bernhardt, B. (2021). BigBrainWarp: Toolbox for integration of BigBrain 3D histology with multimodal neuroimaging. BioRxiv, 2021.05.04.442563. https://doi.org/10.1101/2021.05.04.442563 
Paquola, C., Vos De Wael, R., Wagstyl, K., Bethlehem, R. A. I., Hong, S.-J., Seidlitz, J., Bullmore, E. T., Evans, A. C., Misic, B., Margulies, D. S., Smallwood, J., \& Bernhardt, B. C. (2019). Microstructural and functional gradients are increasingly dissociated in transmodal cortices. PLOS Biology, 17(5), e3000284. https://doi.org/10.1371/journal.pbio.3000284

Park, B., Bethlehem, R. A., Paquola, C., Larivière, S., Rodríguez-Cruces, R., Vos de Wael, R., Neuroscience in Psychiatry Network (NSPN) Consortium, Bullmore, E. T., \& Bernhardt, B. C. (2021). An expanding manifold in transmodal regions characterizes adolescent reconfiguration of structural connectome organization. ELife, 10, e64694. https://doi.org/10.7554/eLife.64694

Park, B., Hong, S.-J., Valk, S. L., Paquola, C., Benkarim, O., Bethlehem, R. A., Di Martino, A., Milham, M. P., Gozzi, A., Yeo, B. T., \& others. (2021). Differences in subcortico-cortical interactions identified from connectome and microcircuit models in autism. Nature Communications, 12(1), 115.

Poldrack, R. A., Baker, C. I., Durnez, J., Gorgolewski, K. J., Matthews, P. M., Munafò, M. R., Nichols, T. E., Poline, J.-B., Vul, E., \& Yarkoni, T. (2017). Scanning the horizon: Towards transparent and reproducible neuroimaging research. Nature Reviews Neuroscience, 18(2), 115-126. https://doi.org/10.1038/nrn.2016.167

Richiardi, J., Altmann, A., Milazzo, A.-C., Chang, C., Chakravarty, M. M., Banaschewski, T., Barker, G. J., Bokde, A. L. W., Bromberg, U., Büchel, C., Conrod, P., Fauth-Bühler, M., Flor, H., Frouin, V., Gallinat, J., Garavan, H., Gowland, P., Heinz, A., Lemaître, H., ... IMAGEN CONSORTIUM. (2015). Correlated gene expression supports synchronous activity in brain networks. Science, 348(6240), 1241-1244. https://doi.org/10.1126/science.1255905

Romme, I. A. C., de Reus, M. A., Ophoff, R. A., Kahn, R. S., \& van den Heuvel, M. P. (2017). Connectome Disconnectivity and Cortical Gene Expression in Patients With Schizophrenia. Biological Psychiatry, 81(6), 495-502. https://doi.org/10.1016/j.biopsych.2016.07.012

Royer, J., Paquola, C., Larivière, S., Vos de Wael, R., Tavakol, S., Lowe, A. J., Benkarim, O., Evans, A. C., Bzdok, D., Smallwood, J., Frauscher, B., \& Bernhardt, B. C. (2020). Myeloarchitecture 
gradients in the human insula: Histological underpinnings and association to intrinsic functional connectivity. NeuroImage, 216, 116859. https://doi.org/10.1016/j.neuroimage.2020.116859

Royer, J., Rodríguez-Cruces, R., Tavakol, S., Larivière, S., Herholz, P., Li, Q., Wael, R. V. de, Paquola, C., Benkarim, O., Park, B., Lowe, A. J., Margulies, D., Smallwood, J., Bernasconi, A., Bernasconi, N., Frauscher, B., \& Bernhardt, B. C. (2021). An Open MRI Dataset for Multiscale Neuroscience. BioRxiv. https://doi.org/10.1101/2021.08.04.454795

Salo, T., Yarkoni, T., Nichols, T. E., Poline, J.-B., Kent, J. D., Gorgolewski, K. J., Glerean, E., Bottenhorn, K. L., Bilgel, M., Wright, J., Reeders, P., Kimbler, A., Nielson, D. N., Yanes, J. A., Pérez, A., Oudyk, K. M., Jarecka, D., \& Laird, A. R. (2020). neurostuff/NiMARE: 0.0.5. Zenodo. https://doi.org/10.5281/zenodo.4408504

Schaefer, A., Kong, R., Gordon, E. M., Laumann, T. O., Zuo, X.-N., Holmes, A. J., Eickhoff, S. B., \& Yeo, B. T. (2017). Local-global parcellation of the human cerebral cortex from intrinsic functional connectivity MRI. Cerebral Cortex, 28(9), 3095-3114. https://doi.org/10.1093/cercor/bhx179

Shine, J. M., Breakspear, M., Bell, P., Ehgoetz, M. K., Shine, R., Koyejo, O., Sporns, O., \& Poldrack, R. (2019). Human cognition involves the dynamic integration of neural activity and neuromodulatory systems. Nat Neurosci, 22(2), 289-296.

Sitek, K. R., Gulban, O. F., Calabrese, E., Johnson, G. A., Lage-Castellanos, A., Moerel, M., Ghosh, S. S., \& De Martino, F. (2019). Mapping the human subcortical auditory system using histology, postmortem MRI and in vivo MRI at 7T. ELife, 8, e48932. https://doi.org/10.7554/eLife.48932

Smith, S. M., Fox, P. T., Miller, K. L., Glahn, D. C., Fox, P. M., Mackay, C. E., Filippini, N., Watkins, K. E., Toro, R., Laird, A. R., \& Beckmann, C. F. (2009). Correspondence of the brain's functional architecture during activation and rest. Proceedings of the National Academy of Sciences, 106(31), 13040-13045. https://doi.org/10.1073/pnas.0905267106

Sormaz, M., Murphy, C., Wang, H., Hymers, M., Karapanagiotidis, T., Poerio, G., Margulies, D. S., Jefferies, E., \& Smallwood, J. (2018). Default mode network can support the level of detail in 
experience during active task states. Proceedings of the National Academy of Sciences, 115(37), $9318-9323$.

Tian, Y., Zalesky, A., Bousman, C., Everall, I., \& Pantelis, C. (2019). Insula Functional Connectivity in Schizophrenia: Subregions, Gradients, and Symptoms. Biological Psychiatry: Cognitive Neuroscience and Neuroimaging, 4(4), 399-408.

Tournier, J. D., Calamante, F., \& Connelly, A. (2012). MRtrix: Diffusion tractography in crossing fiber regions. International Journal of Imaging Systems and Technology, 22(1), 53-66. https://doi.org/10.1002/ima.22005

Truong, W., Minuzzi, L., Soares, C. N., Frey, B. N., Evans, A. C., MacQueen, G. M., \& Hall, G. B. C. (2013). Changes in cortical thickness across the lifespan in major depressive disorder. Psychiatry Research: Neuroimaging, 214(3), 204-211. https://doi.org/10.1016/j.pscychresns.2013.09.003

Valk, S. L., Xu, T., Margulies, D. S., Masouleh, S. K., Paquola, C., Goulas, A., Kochunov, P., Smallwood, J., Yeo, B. T. T., Bernhardt, B. C., \& Eickhoff, S. B. (2020). Shaping Brain Structure: Genetic and Phylogenetic Axes of Macro Scale Organization of Cortical Thickness [Preprint]. Neuroscience. https://doi.org/10.1101/2020.02.10.939561

Van Essen, D. C., Smith, S. M., Barch, D. M., Behrens, T. E. J., Yacoub, E., Ugurbil, K., \& Consortium, W. U.-M. H. C. P. (2013). The WU-Minn Human Connectome Project: An overview. NeuroImage, 80, 62-79. https://doi.org/10.1016/j.neuroimage.2013.05.041

Vogel, J. W., La Joie, R., Grothe, M. J., Diaz-Papkovich, A., Doyle, A., Vachon-Presseau, E., Lepage, C., Vos de Wael, R., Thomas, R. A., Iturria-Medina, Y., Bernhardt, B., Rabinovici, G. D., \& Evans, A. C. (2020). A molecular gradient along the longitudinal axis of the human hippocampus informs large-scale behavioral systems. Nature Communications, 11(1), 960. https://doi.org/10.1038/s41467-020-14518-3

Vos de Wael, R., Benkarim, O., Paquola, C., Lariviere, S., Royer, J., Tavakol, S., Xu, T., Hong, S.-J., Langs, G., Valk, S., \& others. (2020). BrainSpace: A toolbox for the analysis of macroscale gradients in neuroimaging and connectomics datasets. Communications Biology, 3(1), 1-10. 
Vos de Wael, R., Larivière, S., Caldairou, B., Hong, S.-J., Margulies, D. S., Jefferies, E., Bernasconi, A., Smallwood, J., Bernasconi, N., \& Bernhardt, B. C. (2018). Anatomical and microstructural determinants of hippocampal subfield functional connectome embedding. Proceedings of the National Academy of Sciences, 115(40), 10154-10159.

Vos de Wael, R., Royer, J., Tavakol, S., Wang, Y., Paquola, C., Benkarim, O., Eichert, N., Larivière, S., Xu, T., Misic, B., Smallwood, J., Valk, S. L., \& Bernhardt, B. C. (2021). Structural Connectivity Gradients of the Temporal Lobe Serve as Multiscale Axes of Brain Organization and Cortical Evolution. Cerebral Cortex (New York, N.Y.: 1991), 31(11), 5151-5164. https://doi.org/10.1093/cercor/bhab149

Wager, T. D., Lindquist, M., \& Kaplan, L. (2007). Meta-analysis of functional neuroimaging data: Current and future directions. Social Cognitive and Affective Neuroscience, 2(2), 150-158. https://doi.org/10.1093/scan/nsm015

Wagner, H. H., \& Dray, S. (2015). Generating spatially constrained null models for irregularly spaced data using Moran spectral randomization methods. Methods in Ecology and Evolution, 6(10), 11691178. https://doi.org/10.1111/2041-210X.12407

Wagstyl, K., Larocque, S., Cucurull, G., Lepage, C., Cohen, J. P., Bludau, S., Palomero-Gallagher, N., Lewis, L. B., Funck, T., Spitzer, H., Dickscheid, T., Fletcher, P. C., Romero, A., Zilles, K., Amunts, K., Bengio, Y., \& Evans, A. C. (2020). BigBrain 3D atlas of cortical layers: Cortical and laminar thickness gradients diverge in sensory and motor cortices. PLOS Biology, 18(4), e3000678. https://doi.org/10.1371/journal.pbio.3000678

Wang, P., Kong, R., Kong, X., Liégeois, R., Orban, C., Deco, G., Van Den Heuvel, M. P., \& Yeo, B. T. T. (2019). Inversion of a large-scale circuit model reveals a cortical hierarchy in the dynamic resting human brain. Science Advances, 5(1). https://doi.org/10.1126/sciadv.aat7854

Whitaker, K. J., Vértes, P. E., Romero-Garcia, R., Váša, F., Moutoussis, M., Prabhu, G., Weiskopf, N., Callaghan, M. F., Wagstyl, K., Rittman, T., Tait, R., Ooi, C., Suckling, J., Inkster, B., Fonagy, P., Dolan, R. J., Jones, P. B., Goodyer, I. M., Consortium, the N., \& Bullmore, E. T. (2016). 
Adolescence is associated with genomically patterned consolidation of the hubs of the human brain connectome. Proceedings of the National Academy of Sciences, 113(32), 9105-9110. https://doi.org/10.1073/pnas.1601745113

Woo, C.-W., Krishnan, A., \& Wager, T. D. (2014). Cluster-extent based thresholding in fMRI analyses: Pitfalls and recommendations. NeuroImage, $\quad 91, \quad 412-419$. https://doi.org/10.1016/j.neuroimage.2013.12.058

Worsley, K. J., Andermann, M., Koulis, T., MacDonald, D., \& Evans, A. C. (1999). Detecting changes in nonisotropic images. Human Brain Mapping, 8(2-3), 98-101.

Worsley, K. J., Taylor, J. E., Carbonell, F., Chung, M., Duerden, E., Bernhardt, B., Lyttelton, O., Boucher, M., \& Evans, A. (2009). SurfStat: A Matlab toolbox for the statistical analysis of univariate and multivariate surface and volumetric data using linear mixed effects models and random field theory. Neuroimage, 47, S102.

Xu, T., Nenning, K.-H., Schwartz, E., Hong, S.-J., Vogelstein, J. T., Goulas, A., Fair, D. A., Schroeder, C. E., Margulies, D. S., Smallwood, J., Milham, M. P., \& Langs, G. (2020). Cross-species functional alignment reveals evolutionary hierarchy within the connectome. NeuroImage, 223, 117346. https://doi.org/10.1016/j.neuroimage.2020.117346

Yarkoni, T., Poldrack, R. A., Nichols, T. E., Van Essen, D. C., \& Wager, T. D. (2011). Large-scale automated synthesis of human functional neuroimaging data. Nature Methods, 8(8), 665-670. https://doi.org/10.1038/nmeth.1635

Yeo, B. T. T., Krienen, F. M., Sepulcre, J., Sabuncu, M. R., Lashkari, D., Hollinshead, M., Roffman, J. L., Smoller, J. W., Zöllei, L., Polimeni, J. R., Fischl, B., Liu, H., \& Buckner, R. L. (2011). The organization of the human cerebral cortex estimated by intrinsic functional connectivity. $J$. Neurophysiol., 106(3), 1125-1165. https://doi.org/10.1152/jn.00338.2011 


\section{SUPPLEMENTAL FIGURES}

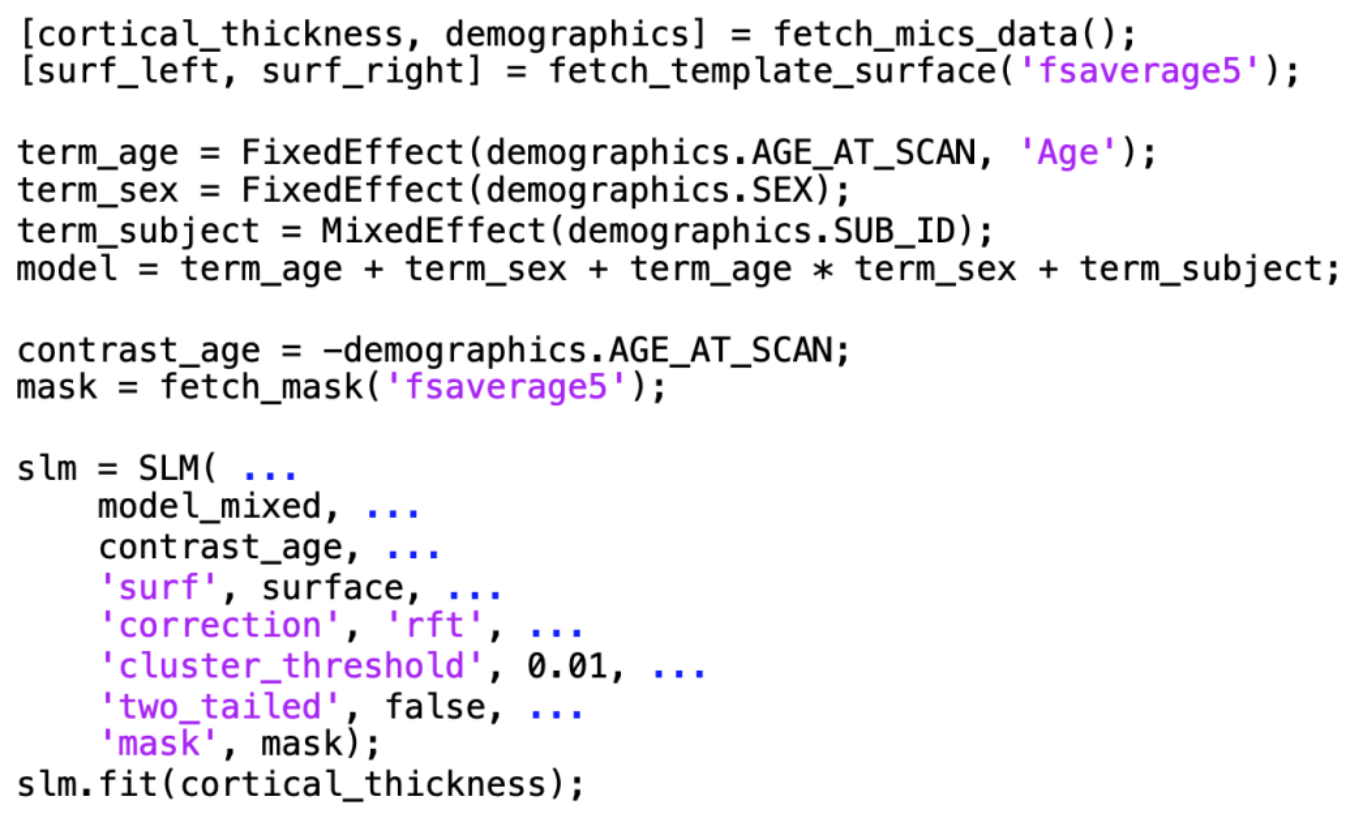

Supplementary Figure 1. MATLAB code for fitting a fixed effect linear model of the effect of age on cortical thickness with BrainStat. 


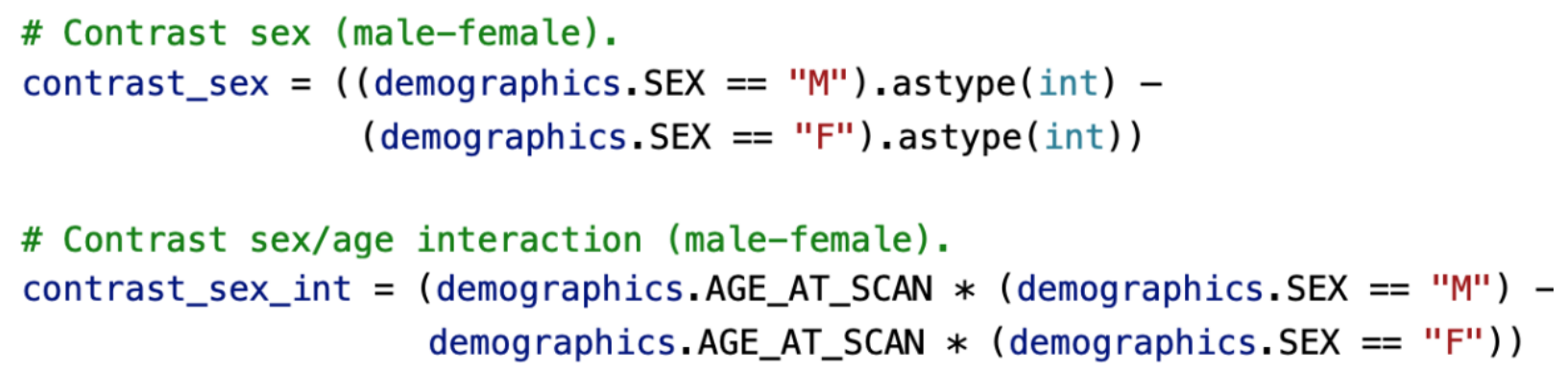

Supplementary Figure 2. Example Python code for contrasts. that probe sex and sex/age interactions, respectively. Equivalent MATLAB code can be found in Supplementary Figure 12. 


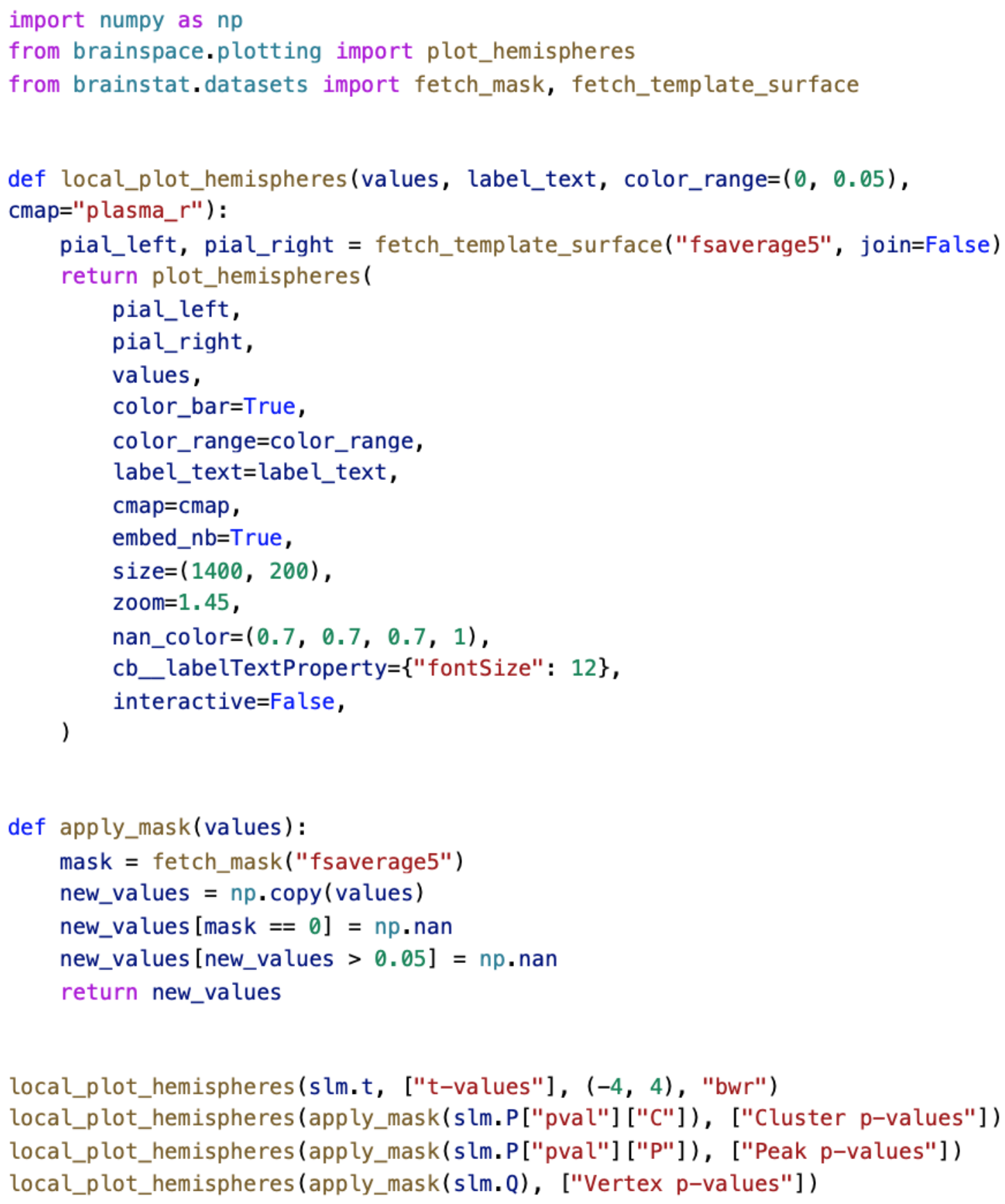

Supplementary Figure 3. Python code to plot the t-values and p-values derived from the code in Figure 1 to the surface. 


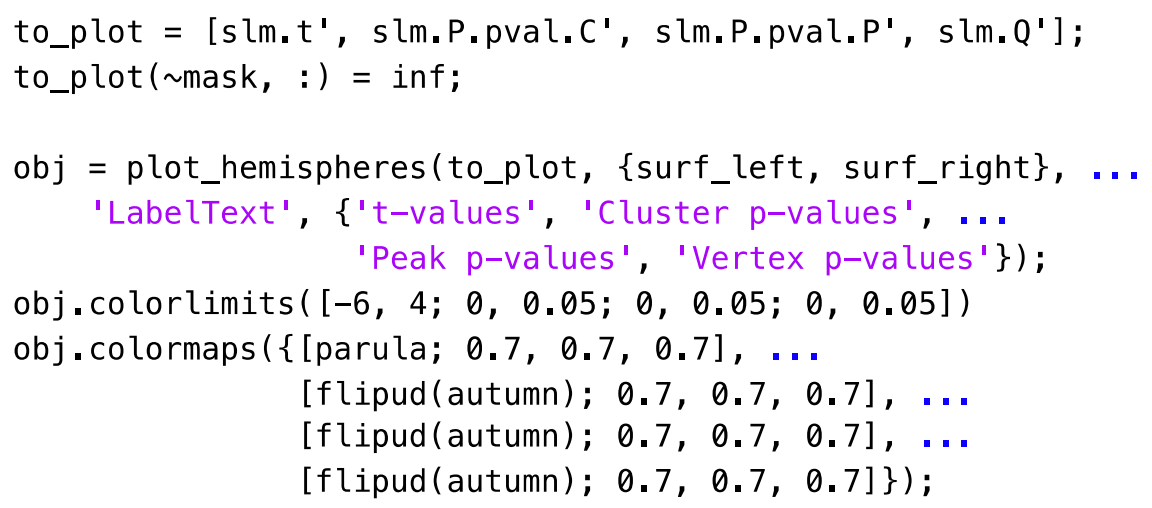

Supplementary Figure 4. MATLAB code to plot the t-values and p-values derived from the code in Supplementary Figure 1 to the surface. 


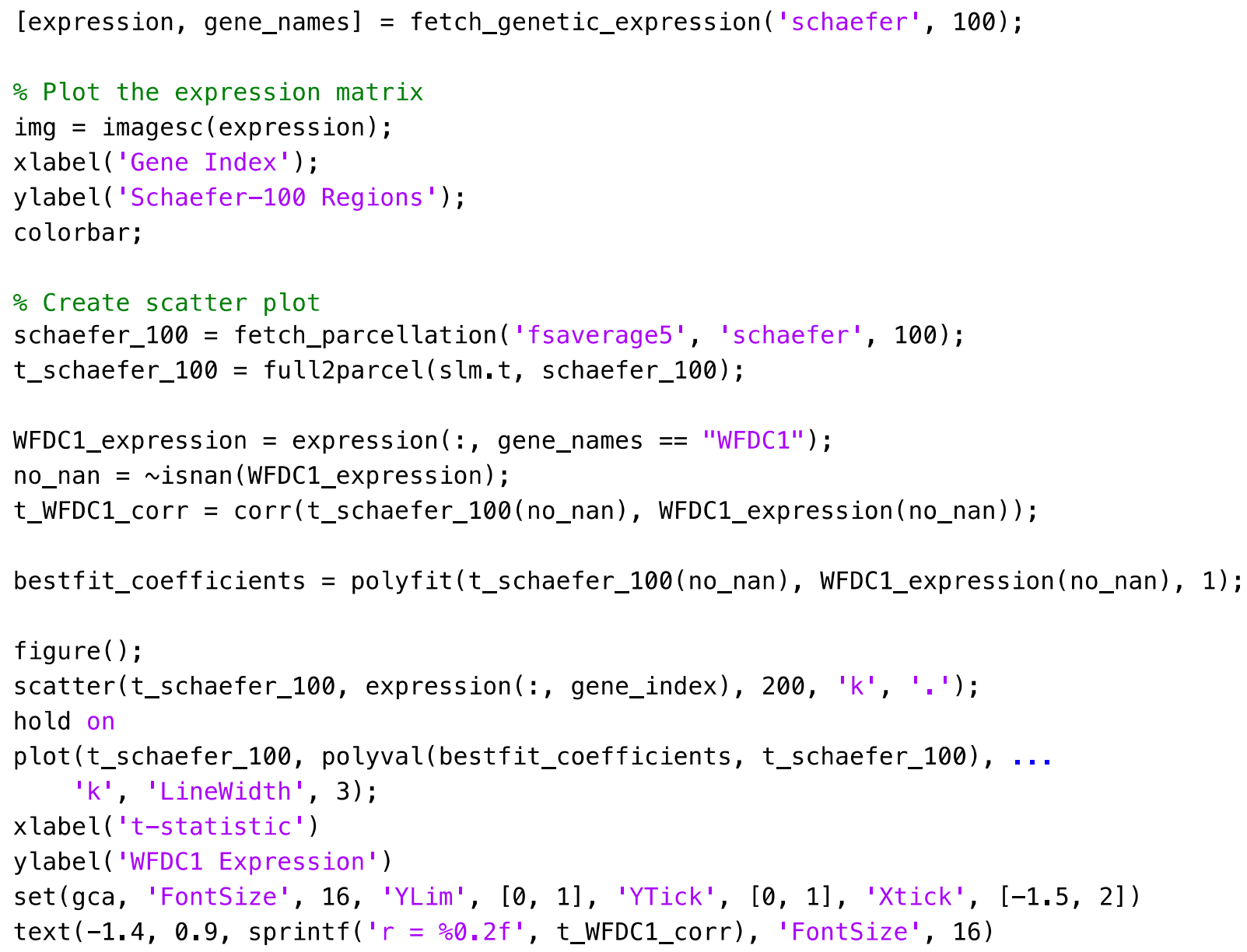

Supplementary Figure 5. Example MATLAB code for computing the genetic expression of a surface parcellation, plotting the matrix, and plotting a scatter plot between the t-statistic computed in Supplementary Figure 1 and the WFDC1 gene expression. 
bioRxiv preprint doi: https://doi.org/10.1101/2022.01.18.476795; this version posted January 25, 2022. The copyright holder for this preprint (which was not certified by peer review) is the author/funder, who has granted bioRxiv a license to display the preprint in perpetuity. It is made available under aCC-BY 4.0 International license.

\section{[correlation, feature] = meta_analytic_decoder(slm.t, 'template', 'fsaverage5'); $\omega c=\operatorname{wordcloud}($ feature $($ correlation $>0)$, correlation (correlation $>0))$;}

Supplementary Figure 6. Example MATLAB code for computing the correlations of the t-statistics computed in Supplementary Figure 1 with meta-analytic analysis of task fMRI studies and plotting these as a word cloud. 


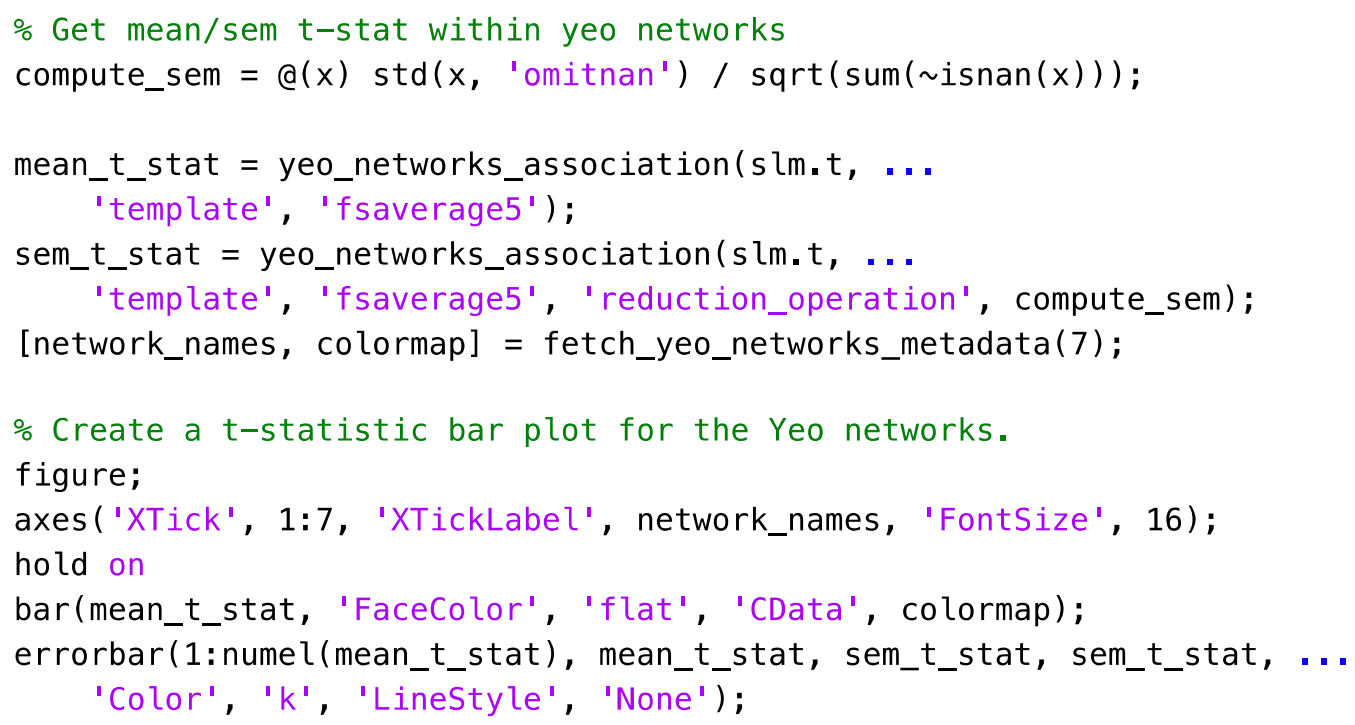

Supplementary Figure 7. Example MATLAB code for computing the correlations of the t-statistics computed in Supplementary Figure 1 with meta-analytic analysis of task fMRI studies and plotting these as a word cloud. 


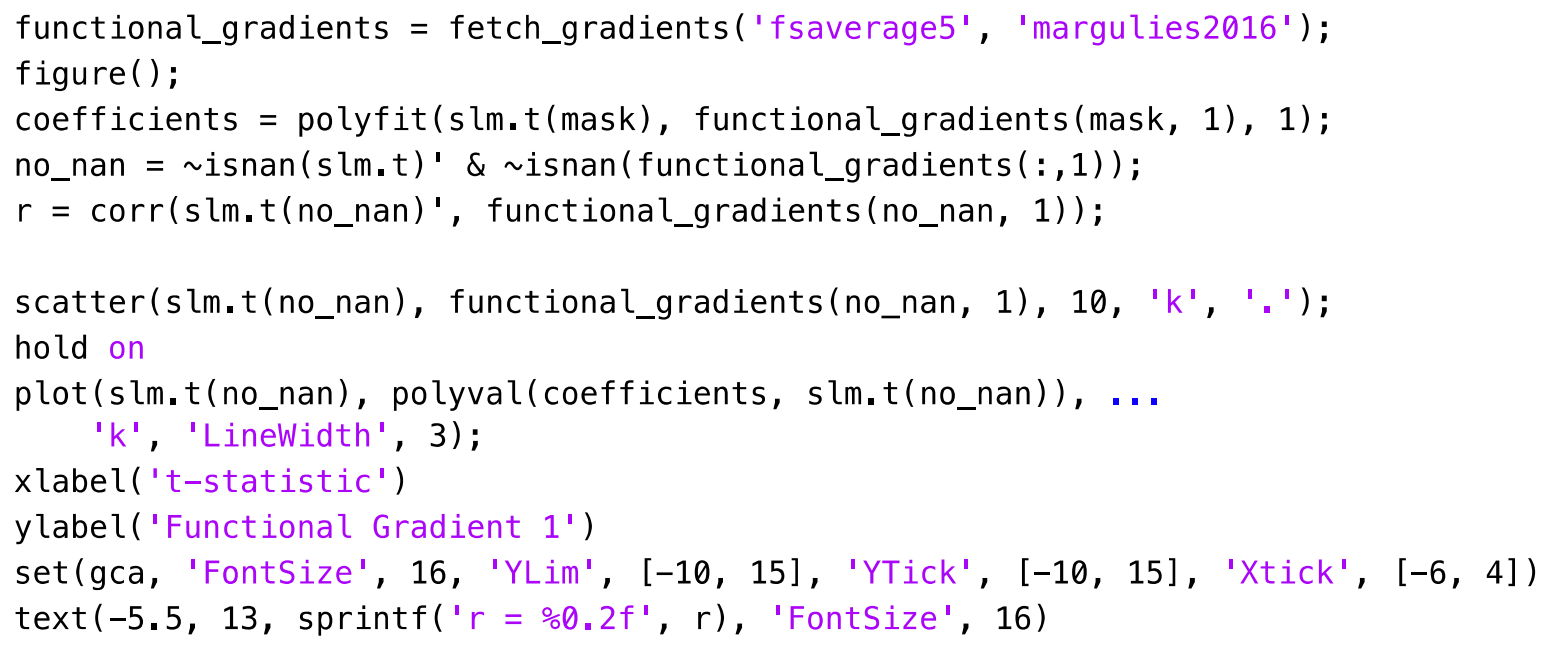

Supplementary Figure 8. Example MATLAB code for computing the correlation of the t-statistic map and the first functional gradient and plotting these in a scatter plot. 


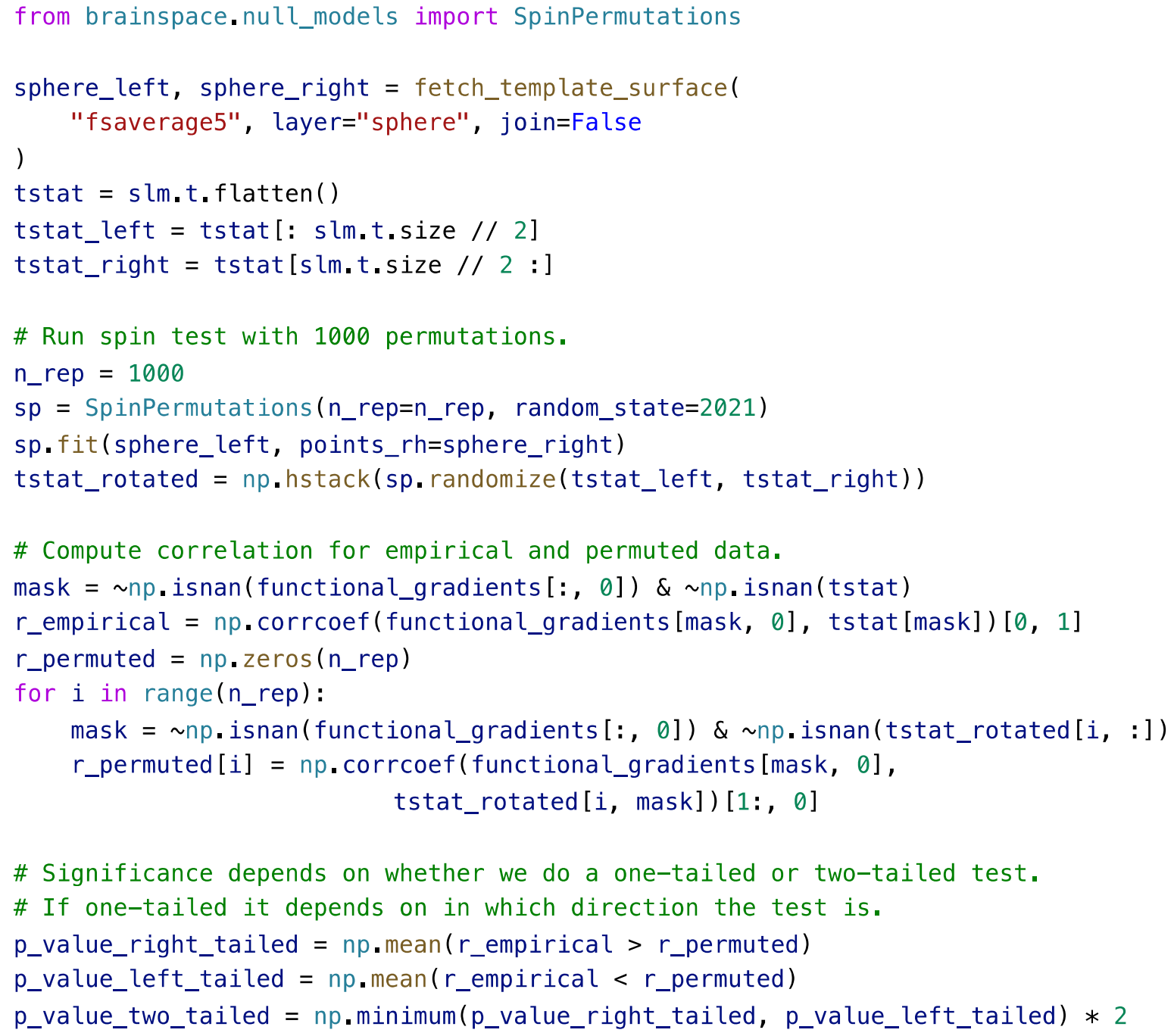

Supplementary Figure 9. Example Python code for computing the significance of the correlation of the t-statistic map and the first functional gradient. 


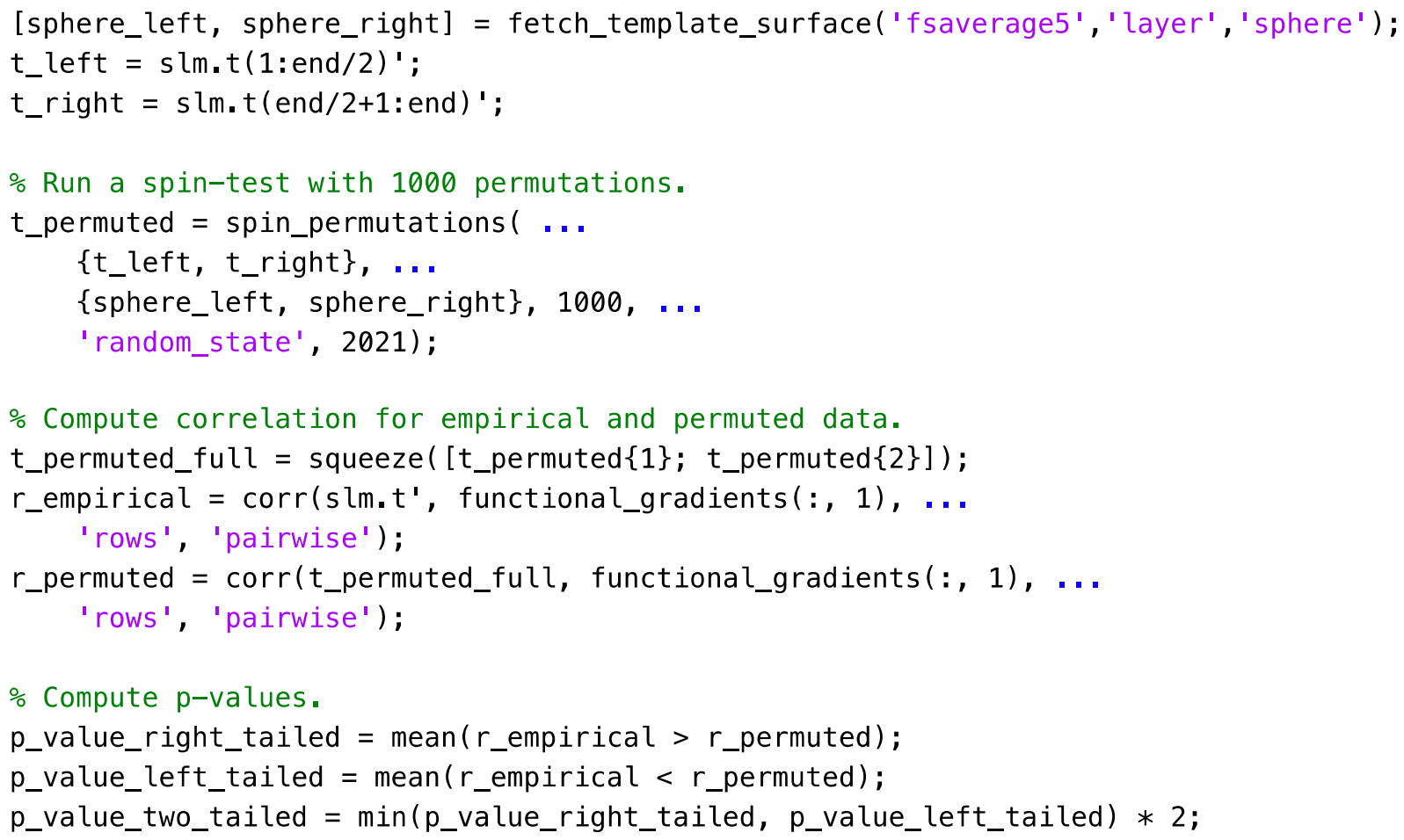

Supplementary Figure 10. Example MATLAB code for computing the significance of the correlation of the t-statistic map and the first functional gradient. 


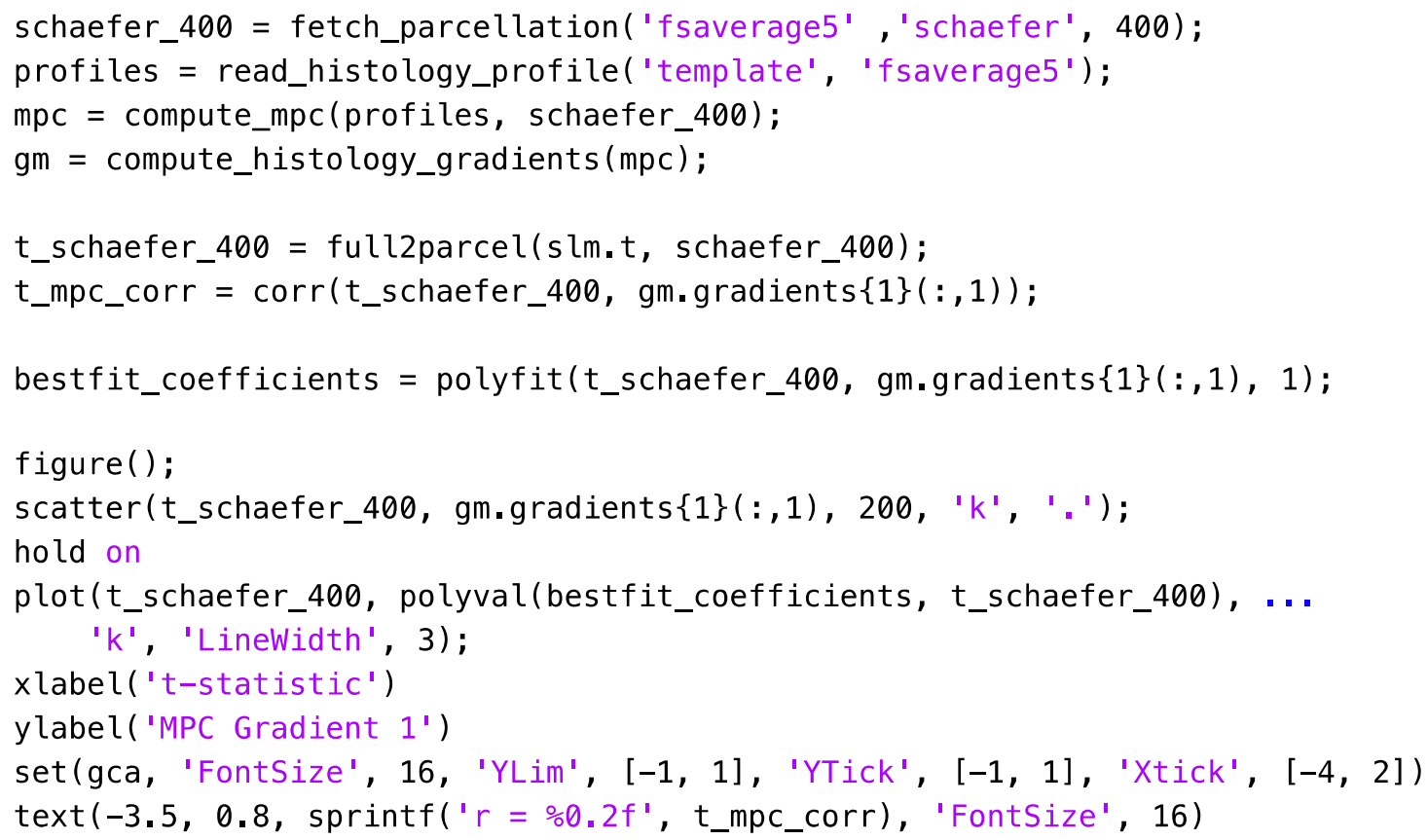

Supplementary Figure 11. Example MATLAB code for computing the correlation of the t-statistic map computed in Supplementary Figure 1 and the first microstructural profile covariance gradient and plotting it in a scatter plot. 
bioRxiv preprint doi: https://doi.org/10.1101/2022.01.18.476795; this version posted January 25, 2022. The copyright holder for this preprint (which was not certified by peer review) is the author/funder, who has granted bioRxiv a license to display the preprint in perpetuity. It is made available under aCC-BY 4.0 International license.

$\%$ Contrast sex (male-female). contrast_sex $=($ demographics.SEX $==$ "M") - (demographics.SEX $==$ "F");

$\%$ Contrast sex/age interaction (male-female). contrast_sex_int $=($ demographics.AGE_AT_SCAN $* *($ demographics.SEX $==" M "))-\ldots$ (demographics.AGE_AT_SCAN $* *($ demographics.SEX $==$ "F"));

Supplementary Figure 12. Example MATLAB code for contrasts that probe sex and sex/age interactions, respectively. 